\title{
The finite element method for MHD flow at high Hartmann numbers
}

\author{
A.I. Nesliturk ${ }^{\mathrm{a}, *}$, M. Tezer-Sezgin ${ }^{\mathrm{b}}$ \\ ${ }^{a}$ Department of Mathematics, Izmir Institute of Technology, 35430 Izmir, Turkey \\ ${ }^{\mathrm{b}}$ Department of Mathematics, Middle East Technical University, 06531 Ankara, Turkey
}

Received 22 January 2004; accepted 8 June 2004

\begin{abstract}
A stabilized finite element method using the residual-free bubble functions (RFB) is proposed for solving the governing equations of steady magnetohydrodynamic duct flow. A distinguished feature of the RFB method is the resolving capability of high gradients near the layer regions without refining mesh. We show that the RFB method is stable by proving that the numerical method is coercive even not only at low values but also at moderate and high values of the Hartmann number. Numerical results confirming theoretical findings are presented for several configurations of interest. The approximate solution obtained by the RFB method is also compared with the analytical solution of Shercliff's problem.
\end{abstract}

(c) 2004 Elsevier B.V. All rights reserved.

Keywords: MHD flow; Stabilized FEM

\section{Introduction}

The problem of the flow of incompressible, viscous, electrically conducting fluids in channels and ducts under a uniform oblique magnetic field is important due to the practical applications in the field of magnetohydrodynamics (MHD). The design of cooling systems with liquid metals for a nuclear reactor, MHD generators, accelerators, pumps and flowmeters are all such applications. The exact solution of the problem can be obtained only for some special cases. Therefore it is important to devise effective numerical methods for the approximate solution of the MHD problem.

\footnotetext{
* Corresponding author.

E-mail address: alinesliturk@iyte.edu.tr (A.I. Nesliturk).
} 
Many researchers investigated the MHD problem in two-dimensional case using several numerical methods. Singh and Lal [5,6] have obtained numerical solutions of the MHD flows through pipes of triangular cross-section using a finite difference method. Then due to the difficulties in fitting arbitrary cross-section of the channel, they presented the finite element method (FEM) solution of channel flows for Hartmann number less than 10 [7,14,8]. Tezer-Sezgin and Koksal extended these studies to moderate Hartmann numbers up to 100 by using the standard FEM with linear and quadratic elements [17]. Further Demendy and Nagy have used the analytical finite element method to obtain their numerical solution in the range of the Hartmann number $M<1000$ in [18]. The boundary element method application of the MHD flow in a rectangular duct with two conducting walls parallel to the applied magnetic field and the other walls being insulators is given by Tezer-Sezgin [16] for Hartmann number up to 10. Solution of MHD flow in a rectangular duct with insulating walls is presented using DQM by Tezer-Sezgin [15] in the presence of an oblique magnetic field. The common deficiency of the existing numerical methods is that they produce physical numerical results in several configurations of interest but Hartmann number $M$ cannot be increased more than 1000. For high Hartmann numbers the difficulty is similar to the advection-diffusion equation when advection process dominates diffusion and can be explained by the formation of layers near the walls or inside the region depending on the boundary conditions.

The present paper uses the Galerkin finite element method with standard piecewise linear polynomials enriched by residual-free bubble (RFB) functions to solve the MHD flow problem in a straight channel of uniform cross-section. The RFB functions enable us to resolve layers without refining the mesh. The RFB method was first proposed by Brezzi and Russo [4] for the advection-diffusion equation. The stability of the method for the advection-diffusion equation has been well investigated for linear and bilinear elements and for a wide range of Peclet number in a series of papers [13,2,12,9]. From a practical point of view, the important range of the Hartmann numbers is $10^{2}<M<10^{6}$. Using the RFB method we are able to compute accurate numerical approximations to the solution of the MHD flow problem for this range of the Hartmann number. Computational power of the new numerical method can be explained by its stability analysis. This will be done at moderate and high values of Hartmann number for triangular elements. We then proceed numerical experiments that show robustness of the numerical method.

The paper is organized as follows. In Section 2 the governing equations of the MHD flow problem under consideration are specified. In Section 3 we discuss the discretization procedure with an emphasis on the description of the residual-free bubble method applied to the MHD problem. The stability of the numerical method for moderate and high Hartmann numbers is investigated in Section 4. Some computational issues that makes computer implementation more convenient are also discussed in this section. Finally the computational power of the RFB method is tested via a comparison with the analytical solution of Shercliff's problem in Section 5. Further numerical results verifying robustness of the method are also presented.

\section{Definition of the problem}

We consider the problem of finding the velocity $V$ and the induced magnetic field $B$ for a laminar, fully developed flow of an incompressible, viscous, electrically conducting fluid in a straight channel of uniform cross-section. The fluid is driven down by a constant pressure gradient, the direction of the uniform transverse applied magnetic field $B_{0}$ may be arbitrary to the $x$-axis, and the fields $V$ and $B$ are parallel to the $z$-axis. The dimensionless governing equations of this model [11]

$$
\begin{aligned}
& \epsilon \nabla^{2} V+\tilde{a} \cdot \nabla B=-\epsilon \quad \text { in } \Omega, \\
& \epsilon \nabla^{2} B+\tilde{a} \cdot \nabla V=0 \quad \text { in } \Omega,
\end{aligned}
$$




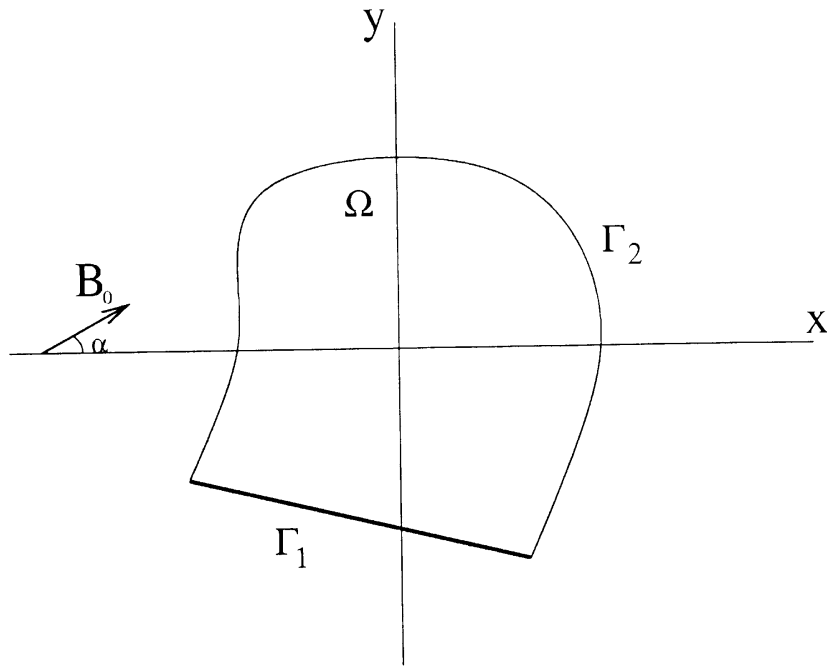

Fig. 1. A 2D duct flow.

where $\Omega$ is a bounded domain in $\mathbb{R}^{2}$ with the boundary $\partial \Omega$ and $\tilde{a}=(\sin \alpha, \cos \alpha) ; \alpha$ is the angle between the externally applied magnetic field and the $x$-axis (see Fig. 1). The parameter $\epsilon$ is the inverse of the Hartmann number $\epsilon=1 / M$ where this number is defined by $M=B_{0} L(\delta / \mu)^{1 / 2} ; B_{0}$ is the intensity of the external magnetic field, $L$ is the characteristic dimension of the channel cross-section, $\delta$ and $\mu$ are the electric conductivity and coefficient of viscosity of the fluid respectively. The general boundary conditions which are suitable in practice for the MHD problem can be expressed as

$$
\begin{array}{ll}
V=0 & \text { on } \partial \Omega, \\
B=0 & \text { on } \Gamma_{1}, \\
\frac{\partial B}{\partial n}=0 & \text { on } \Gamma_{2},
\end{array}
$$

where $\partial \Omega=\Gamma_{1} \cup \Gamma_{2}$ with $\Gamma_{1} \cap \Gamma_{2}=\emptyset$. We call $\Gamma_{1}$ as the insulated part and $\Gamma_{2}$ as the conducting part of the boundary $\partial \Omega$. We use standard notation for function spaces: $C^{0}(\Omega)$ is the space of continuous functions in $\Omega, L^{2}(\Omega)$ is the space of square-integrable functions in $\Omega, H^{1}(\Omega)$ is the Sobolev space of $L^{2}(\Omega)$ functions whose derivatives are square-integrable functions in $\Omega$ and $H_{0}^{1}(\Omega)$ is the Sobolev space of $H^{1}(\Omega)$ functions in $\Omega$ with zero value on the boundary $\partial \Omega$. Also $(\cdot, \cdot)$ denotes the $L_{2}$ inner product on $\Omega$.

\section{The numerical method}

Let $\mathscr{V} \times \mathscr{B}=H_{0}^{1}(\Omega) \times H^{1}(\Omega)$. The problem (1) with boundary conditions (2) can be equivalently stated as the following variational problem: Find $V \in \mathscr{V}$ and $B \in \mathscr{B}$ such that

$$
c(V, B ; \widetilde{V}, \widetilde{B})=(-\epsilon, \widetilde{V}) \text { for all }(\widetilde{V}, \widetilde{B}) \in \mathscr{V} \times \mathscr{B},
$$

where

$$
c(V, B ; \widetilde{V}, \widetilde{B})=-\epsilon(\nabla V, \nabla \widetilde{V})+(\tilde{a} \cdot \nabla B, \widetilde{V})-\epsilon(\nabla B, \nabla \widetilde{B})+(\tilde{a} \cdot \nabla V, \widetilde{B}) .
$$


To specify Galerkin finite element method we choose a partition $\mathscr{K}$ of $\Omega$ consisting of triangular elements in the standard way (e.g. no overlapping, no vertex on the edge of neighboring element). Let $\mathscr{V}_{h} \times \mathscr{B}_{h}$ denote the finite dimensional subspace of $\mathscr{V} \times \mathscr{B}$. We assume $\mathscr{V}_{h}, \mathscr{B}_{h}$ consist of typical $C^{0}(\Omega)$ finite element functions and that if $B_{h} \in \mathscr{B}_{h}$ then $B_{h}(x)=0$ for any $x$ belonging to the part $\Gamma_{1}$ of the boundary.

The finite dimensional subspaces that we wish to work are given by

$$
\begin{aligned}
& \mathscr{V}_{h}=\mathscr{V}_{1}+\mathscr{V}_{\mathrm{b}}=\mathscr{V}_{1} \oplus\left(\bigcup_{K} \mathscr{B}_{V}(K)\right) \subset \mathscr{V}=H_{0}^{1}(\Omega), \\
& \mathscr{B}_{h}=\mathscr{B}_{1}+\mathscr{B}_{\mathrm{b}}=\mathscr{B}_{1} \oplus\left(\bigcup_{K} \mathscr{B}_{M}(K)\right) \subset \mathscr{B}=H^{1}(\Omega),
\end{aligned}
$$

where $\mathscr{V}_{1}$ and $\mathscr{B}_{1}$ denote the finite element spaces of continuous, piecewise linear polynomials defined over triangular elements, $\mathscr{B}_{V}(K) \subset H_{0}^{1}(K)$ and $\mathscr{B}_{M}(K) \subset H_{0}^{1}(K)$. Moreover the finite dimensional spaces $\bigcup_{K} \mathscr{B}_{V}(K)$ and $\bigcup_{K} \mathscr{B}_{M}(K)$ are spanned by so-called residual- free bubble functions which will be specified later. We note that the spaces $\mathscr{V}_{1}$ and $\mathscr{V}_{\mathrm{b}}$ are orthogonal to each other with respect to the inner product $(\nabla ., \nabla$.$) , which is also true for the pair of spaces \mathscr{B}_{1}$ and $\mathscr{B}_{\mathrm{b}}$. To prove the orthogonality of the spaces $\mathscr{B}_{1}$ and $\mathscr{B}_{\mathrm{b}}$ observe

$$
\left(\nabla B_{1}, \nabla B_{\mathrm{b}}\right)=\sum_{K}\left(\nabla B_{1}, \nabla B_{\mathrm{b}}\right)_{K}=\sum_{K}\left\{\int_{\partial K} \frac{\partial B_{1}}{\partial n} B_{\mathrm{b}} \mathrm{d} \Gamma-\int_{K} B_{\mathrm{b}} \Delta B_{1} \mathrm{~d} \Omega\right\}=0,
$$

since $B_{\mathrm{b}}=0$ on $\partial K$ and $\Delta B_{1}=0$ on each element $K$. Obviously the subscript $K$ indicates that integration is restricted to the element $K$.

Let us state the standard Galerkin finite element method for the problem (3) with our choice of finite dimensional spaces: Find $V_{h} \in \mathscr{V}_{h}$ and $B_{h} \in \mathscr{B}_{h}$. such that

$$
c_{h}\left(V_{h}, B_{h} ; \widetilde{V}_{h}, \widetilde{B}_{h}\right)=\left(-\epsilon, \widetilde{V}_{h}\right) \text { for all }\left(\widetilde{V}_{h}, \widetilde{B}_{h}\right) \in \mathscr{V}_{h} \times \mathscr{B}_{h},
$$

where

$$
c_{h}\left(V_{h}, B_{h} ; \widetilde{V}_{h}, \widetilde{B}_{h}\right)=-\epsilon\left(\nabla V_{h}, \nabla \widetilde{V}_{h}\right)+\left(\tilde{a} \cdot \nabla B_{h}, \widetilde{V}_{h}\right)-\epsilon\left(\nabla B_{h}, \nabla \widetilde{B}_{h}\right)+\left(\tilde{a} \cdot \nabla V_{h}, \widetilde{B}_{h}\right) .
$$

Bubble functions are required to vanish on the boundary $\partial K$ of each element $K$ by definition. For the particular case of the residual-free bubbles, we define the bubble component $V_{\mathrm{b}}$ of $V_{h}$ and $B_{\mathrm{b}}$ of $B_{h}$ by also requiring that the pair $\left\{V_{h}, B_{h}\right\}$ satisfy the original differential equation (1) in the interior of each $K$ and zero elsewhere. That is

$$
\begin{aligned}
& \epsilon \nabla^{2}\left(V_{1}+V_{\mathrm{b}}\right)+\tilde{a} \cdot \nabla\left(B_{1}+B_{\mathrm{b}}\right)=-\epsilon \text { in } K, \\
& \epsilon \nabla^{2}\left(B_{1}+B_{\mathrm{b}}\right)+\tilde{a} \cdot \nabla\left(V_{1}+V_{\mathrm{b}}\right)=0 \quad \text { in } K, \\
& V_{\mathrm{b}}=B_{\mathrm{b}}=0 \text { on } \partial K .
\end{aligned}
$$

In particular this implies, on a mesh of triangles, that for each $K$ we have

$$
\begin{aligned}
& \epsilon \nabla^{2} V_{\mathrm{b}}+\tilde{a} \cdot \nabla B_{\mathrm{b}}=-\epsilon-\tilde{a} \cdot \nabla B_{1} \quad \text { in } K, \\
& \epsilon \nabla^{2} B_{\mathrm{b}}+\tilde{a} \cdot \nabla V_{\mathrm{b}}=-\tilde{a} \cdot \nabla V_{1} \quad \text { in } K, \\
& V_{\mathrm{b}}=B_{\mathrm{b}}=0 \quad \text { on } \partial K .
\end{aligned}
$$

In (5), take $\widetilde{V}_{h}=\widetilde{V}_{\mathrm{b}}$ and $\widetilde{B}_{h}=\widetilde{B}_{\mathrm{b}}$ on $K$ and $\widetilde{V}_{h}=\widetilde{B}_{h}=0$ elsewhere to obtain

$$
c_{h}\left(V_{h}, B_{h} ; \widetilde{V}_{\mathrm{b}}, \widetilde{B}_{\mathrm{b}}\right)_{K}=\left(-\epsilon, \widetilde{V}_{\mathrm{b}}\right)_{K} .
$$


Our choice of the residual-free bubbles in Eq. (6) ensures that Eq. (8) is satisfied automatically. Then the numerical method that we implement is obtained by setting $\widetilde{V}_{h}=\widetilde{V}_{1}$ and $\widetilde{B}_{h}=\widetilde{B}_{1}$ in Eq. (5):

$$
c_{h}\left(V_{h}, B_{h} ; \widetilde{V}_{1}, \widetilde{B}_{1}\right)=\left(-\epsilon, \widetilde{V}_{1}\right) \text { for all }\left(\widetilde{V}_{1}, \widetilde{B}_{1}\right) \in \mathscr{V}_{1} \times \mathscr{B}_{1}
$$

where

$$
\begin{aligned}
& c_{h}\left(V_{h}, B_{h} ; \widetilde{V}_{1}, \widetilde{B}_{1}\right)=c_{h}\left(V_{1}, B_{1} ; \widetilde{V}_{1}, \widetilde{B}_{1}\right)+c_{h}\left(V_{\mathrm{b}}, B_{\mathrm{b}} ; \widetilde{V}_{1}, \widetilde{B}_{1}\right), \\
& c_{h}\left(V_{1}, B_{1} ; \widetilde{V}_{1}, \widetilde{B}_{1}\right)=-\epsilon\left(\nabla V_{1}, \nabla \widetilde{V}_{1}\right)+\left(a \cdot \nabla B_{1}, \widetilde{V}_{1}\right)-\epsilon\left(\nabla B_{1}, \nabla \widetilde{B}_{1}\right)+\left(a \cdot \nabla V_{1}, \widetilde{B}_{1}\right), \\
& c_{h}\left(V_{\mathrm{b}}, B_{\mathrm{b}} ; \widetilde{V}_{1}, \widetilde{B}_{1}\right)=\left(a \cdot \nabla B_{\mathrm{b}}, \widetilde{V}_{1}\right)+\left(a \cdot \nabla V_{\mathrm{b}}, \widetilde{B}_{1}\right),
\end{aligned}
$$

or, by integration by parts,

$$
c_{h}\left(V_{\mathrm{b}}, B_{\mathrm{b}} ; \widetilde{V}_{1}, \widetilde{B}_{1}\right)=-\left(B_{\mathrm{b}}, \tilde{a} \cdot \nabla \widetilde{V}_{1}\right)-\left(V_{\mathrm{b}}, \tilde{a} \cdot \nabla \widetilde{B}_{1}\right),
$$

where bubble functions $\left\{V_{\mathrm{b}}, B_{\mathrm{b}}\right\}$ are defined in terms of $\left\{V_{1}, B_{1}\right\}$ by Eq. (7). Thus the enrichment of the finite element spaces of piecewise linear functions by bubble functions can be viewed as a modification of the Galerkin formulation by the addition of two additional terms: $\left(a \cdot \nabla B_{\mathrm{b}}, \widetilde{V}_{1}\right)$ and $\left(a \cdot \nabla V_{\mathrm{b}}, \widetilde{B}_{1}\right)$.

\section{Stability of the numerical method}

We will show that additional terms are actually responsible for the stability of the numerical method, especially when the Hartman number is high. We do that by proving that the form $c_{h}\left(V_{h}, B_{h} ; \widetilde{V}_{1}, \widetilde{B}_{1}\right)$ is coercive over the product space $\mathscr{V}_{h} \times \mathscr{B}_{h} \times \mathscr{V}_{1} \times \mathscr{B}_{1}$ under the assumption that the triangulation is regular and this regularity is uniform as $h \rightarrow 0$.

For large values of $\epsilon$, the form $c_{h}$ is coercive over $\mathscr{V}_{h} \times \mathscr{B}_{h} \times \mathscr{V}_{1} \times \mathscr{B}_{1}$ because the bubble functions becomes negligible and the sum of advection terms are zero by integration by parts. Thus we derive the stability estimates for small values of $\varepsilon$. We will do this in two separate cases in the following subsections.

A linear change of variables decouples Eq. (1) into a pair of advection-diffusion problems in the case that the boundary $\partial \Omega$ is perfectly insulated $(B=0)$. When $\epsilon$ is small the advection terms become dominant and it is appropriate to use a stabilized FEM, for example, the residual-free bubble method. The stability of the residual-free bubble method for the advection-diffusion problems was investigated for triangular elements in [12,3]. By using some basic arguments of [12] we prove that the form $c_{h}$ is coercive over $\mathscr{V}_{h} \times \mathscr{B}_{h} \times \mathscr{V}_{1} \times \mathscr{B}_{1}$ resulting in the numerical method (9) is stable.

\subsection{The asymptotical case: $\epsilon \rightarrow 0$}

Since the bubble functions are required to vanish over the whole element boundary $\partial K$, the change of variables

$$
\begin{aligned}
& U_{\mathrm{b}}^{1}=V_{\mathrm{b}}+B_{\mathrm{b}}, \\
& U_{\mathrm{b}}^{2}=V_{\mathrm{b}}-B_{\mathrm{b}},
\end{aligned}
$$

decouples Eq. (7) into a pair of advection-diffusion equation admitting the advection in opposite directions:

$$
\begin{array}{ll}
\epsilon \nabla^{2} U_{\mathrm{b}}^{1}+\tilde{a} \cdot \nabla U_{\mathrm{b}}^{1}=-\epsilon-\tilde{a} \cdot \nabla B_{1}-\tilde{a} \cdot \nabla V_{1} & \text { in } K, \\
\epsilon \nabla^{2} U_{\mathrm{b}}^{2}-\tilde{a} \cdot \nabla U_{\mathrm{b}}^{2}=-\epsilon-\tilde{a} \cdot \nabla B_{1}+\tilde{a} \cdot \nabla V_{1} & \text { in } K, \\
U_{\mathrm{b}}^{1}=U_{\mathrm{b}}^{2}=0 \quad \text { on } \partial K . &
\end{array}
$$


For each element $K$, let $\partial K_{1}=\{x \in \partial K: \tilde{a} \cdot \tilde{n}(x)<0\}$ be the first part of its boundary and $\partial K_{2}=\{x \in \partial K: \tilde{a} \cdot \tilde{n}(x)>0\}$ be the second part of its boundary, where $\tilde{n}$ is the outward-pointing unit normal to $\partial K$. Assuming that $\tilde{a} \cdot \tilde{n}(x)$ is bounded away from zero; then excluding vertices, for each $K$ we have $\partial K=\partial K_{1} \cup \partial K_{2}$.

Let $\widehat{U}_{\mathrm{b}}^{1}$ and $\widehat{U}_{\mathrm{b}}^{2}$ be the asymptotical solution of Eq. (12). That is, for each $K$, we have,

$$
\begin{aligned}
& \tilde{a} \cdot \nabla \widehat{U}_{\mathrm{b}}^{1}=-\tilde{a} \cdot \nabla B_{1}-\tilde{a} \cdot \nabla V_{1} \quad \text { in } K, \quad \widehat{U}_{\mathrm{b}}^{1}=0 \quad \text { on } \partial K_{2}, \\
& -\tilde{a} \cdot \nabla \widehat{U}_{\mathrm{b}}^{2}=-\tilde{a} \cdot \nabla B_{1}+\tilde{a} \cdot \nabla V_{1} \quad \text { in } K, \quad \widehat{U}_{\mathrm{b}}^{2}=0 \quad \text { on } \partial K_{1} .
\end{aligned}
$$

Let $\left(x_{P}, x_{Q}\right)$ be a line segment that is parallel to the direction of the vector $\tilde{a}$ and that lies in a single $K$ with $x_{P} \in \partial K_{1}$ and $x_{Q} \in \partial K_{2}$ (Fig. 2). Then

$$
\begin{aligned}
& \widehat{U}_{\mathrm{b}}^{1}(x)=\frac{1}{|\tilde{a}|} \int_{x}^{x_{Q}} \tilde{a} \cdot \nabla\left(B_{1}+V_{1}\right) \mathrm{d} s, \\
& \widehat{U}_{\mathrm{b}}^{2}(x)=\frac{1}{|\tilde{a}|} \int_{x_{P}}^{x} \tilde{a} \cdot \nabla\left(B_{1}-V_{1}\right) \mathrm{d} s,
\end{aligned}
$$

for $x \in\left(x_{P}, x_{Q}\right)$ where $s$ is the arc length parameter. Hence recalling (11) we have

$$
\begin{aligned}
& \widehat{V}_{\mathrm{b}}=\frac{\widehat{U}_{\mathrm{b}}^{1}+\widehat{U}_{\mathrm{b}}^{2}}{2}=\frac{1}{2|\tilde{a}|} \int_{x}^{x_{Q}} \tilde{a} \cdot \nabla\left(B_{1}+V_{1}\right) \mathrm{d} s+\int_{x_{P}}^{x} \tilde{a} \cdot \nabla\left(B_{1}-V_{1}\right) \mathrm{d} s, \\
& \widehat{B}_{\mathrm{b}}=\frac{\widehat{U}_{\mathrm{b}}^{1}-\widehat{U}_{\mathrm{b}}^{2}}{2}=\frac{1}{2|\tilde{a}|} \int_{x}^{x_{Q}} \tilde{a} \cdot \nabla\left(B_{1}+V_{1}\right) \mathrm{d} s-\int_{x_{P}}^{x} \tilde{a} \cdot \nabla\left(B_{1}-V_{1}\right) \mathrm{d} s .
\end{aligned}
$$

Letting $\widetilde{V}_{1}=-V_{1}, \widetilde{B}_{1}=-B_{1}$ and plugging the expressions for $\widehat{V}_{\mathrm{b}}$ and $\widehat{B}_{\mathrm{b}}$ above into the terms containing bubble functions (10) in the discrete problem (9) we get
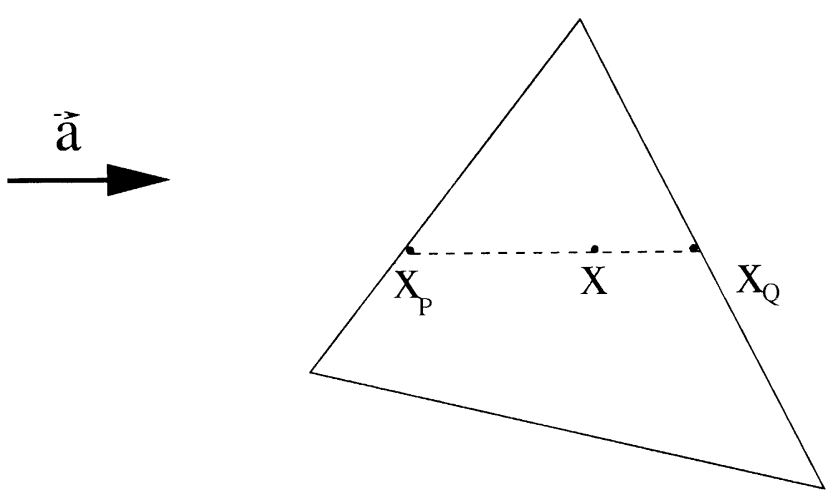

Fig. 2. The definition of the line segment $\left(x_{P}, x_{Q}\right)$. 


$$
\begin{aligned}
-\left(\widehat{B}_{\mathrm{b}}, \tilde{a} \cdot \nabla \widetilde{V}_{1}\right)_{K}-\left(\widehat{V}_{\mathrm{b}}, \tilde{a} \cdot \nabla \widetilde{B}_{1}\right)_{K}= & \left.\frac{1}{|\tilde{a}|}\left(\tilde{a} \cdot \nabla\left(B_{1}+V_{1}\right)\right)^{2}\right|_{K}\left(\int_{x}^{x_{Q}} \mathrm{~d} s, 1\right)_{K} \\
& +\left.\frac{1}{|\tilde{a}|}\left(\tilde{a} \cdot \nabla\left(B_{1}-V_{1}\right)\right)^{2}\right|_{K}\left(\int_{x_{P}}^{x} \mathrm{~d} s, 1\right)_{K} \\
= & \frac{h_{a}}{3|\tilde{a}|}\left\|\tilde{a} \cdot \nabla\left(B_{1}+V_{1}\right)\right\|_{K}^{2}+\frac{h_{a}}{3|\tilde{a}|}\left\|\tilde{a} \cdot \nabla\left(B_{1}-V_{1}\right)\right\|_{K}^{2} \quad \text { (see [1]) } \\
= & \frac{2 h_{a}}{3|\tilde{a}|}\left\|\tilde{a} \cdot \nabla V_{1}\right\|_{K}^{2}+\frac{2 h_{a}}{3|\tilde{a}|}\left\|\tilde{a} \cdot \nabla B_{1}\right\|_{K}^{2} \quad \text { (Parallelogram law) }
\end{aligned}
$$

since all $\tilde{a} \cdot \nabla$ terms are constant. That shows the extra terms (10) in the numerical method (9) adds stability through the terms (15). Here $h_{a}$ is defined as the longest line segment parallel to the vector $\tilde{a}$ and contained in the element $K$.

Remark 1. In computer implementation, we go back to Eq. (14) and evaluate the integral so that we get

$$
\begin{aligned}
& \widehat{U}_{\mathrm{b}}^{1}(x)=V_{1}\left(x_{Q}\right)+B_{1}\left(x_{Q}\right)-V_{1}(x)-B_{1}(x), \\
& \widehat{U}_{\mathrm{b}}^{2}(x)=B_{1}(x)-V_{1}(x)-B_{1}\left(x_{P}\right)+V_{1}\left(x_{P}\right) .
\end{aligned}
$$

Corresponding values of $\widehat{V}_{\mathrm{b}}$ and $\widehat{B}_{\mathrm{b}}$, can be found by transforming variables back in (11):

$$
\begin{aligned}
& \widehat{V}_{\mathrm{b}}^{1}(x)=\frac{V_{1}\left(x_{Q}\right)+V_{1}\left(x_{P}\right)}{2}+\frac{B_{1}\left(x_{Q}\right)-B_{1}\left(x_{P}\right)}{2}-V_{1}(x), \\
& \widehat{B}_{\mathrm{b}}^{2}(x)=\frac{V_{1}\left(x_{Q}\right)-V_{1}\left(x_{P}\right)}{2}+\frac{B_{1}\left(x_{Q}\right)+B_{1}\left(x_{P}\right)}{2}-B_{1}(x) .
\end{aligned}
$$

Now plugging these expressions into the terms containing the bubbles (10) in the numerical method (9) we get

$$
\begin{aligned}
-\left(\widehat{B}_{\mathrm{b}}, \tilde{a} \cdot \nabla \widetilde{V}_{1}\right)_{K}-\left(\widehat{V}_{\mathrm{b}}, \tilde{a} \cdot \nabla \widetilde{B}_{1}\right)_{K}= & -\left(\frac{V_{1}\left(x_{Q}\right)-V_{1}\left(x_{P}\right)}{2}, \tilde{a} \cdot \nabla \widetilde{V}_{1}\right)_{K} \\
& -\left(\frac{B_{1}\left(x_{Q}\right)+B_{1}\left(x_{P}\right)}{2}, \tilde{a} \cdot \nabla \widetilde{V}_{1}\right)_{K}+\left(B_{1}, \tilde{a} \cdot \nabla \widetilde{V}_{1}\right)_{K} \\
& -\left(\frac{V_{1}\left(x_{Q}\right)+V_{1}\left(x_{P}\right)}{2}, \tilde{a} \cdot \nabla \widetilde{B}_{1}\right)_{K} \\
& -\left(\frac{B_{1}\left(x_{Q}\right)-B_{1}\left(x_{P}\right)}{2}, \tilde{a} \cdot \nabla \widetilde{B}_{1},\right)_{K}+\left(V_{1}, \tilde{a} \cdot \nabla \widetilde{B}_{1}\right)_{K}
\end{aligned}
$$

The terms containing the functions whose values are determined by the values of interpolation functions on the element boundary are the additional terms to the terms found in the Galerkin formulation with piecewise linears; it is these additional terms that yield stability in the numerical method.

\subsection{Midrange: $0<\epsilon \ll 1$}

We can further obtain a stability estimate for the numerical method for the values of $\epsilon$ in the range $0<\epsilon \ll 1$. Let us fix an element $K$. Decompose the bubble functions $V_{\mathrm{b}}$ and $B_{\mathrm{b}}$ as $V_{\mathrm{b}}=V_{\mathrm{b} 0}+V_{\mathrm{b}}^{\epsilon}$ and $B_{\mathrm{b}}=B_{\mathrm{b} 0}+B_{\mathrm{b}}^{\epsilon}$ where they are defined, on each $K$, by 


$$
\begin{aligned}
& \epsilon \nabla^{2} V_{\mathrm{b} 0}+\tilde{a} \cdot \nabla B_{\mathrm{b} 0}=-\tilde{a} \cdot \nabla B_{1} \quad \text { in } K, \\
& \epsilon \nabla^{2} B_{\mathrm{b} 0}+\tilde{a} \cdot \nabla V_{\mathrm{b} 0}=-\tilde{a} \cdot \nabla V_{1} \quad \text { in } K, \\
& V_{\mathrm{b} 0}=B_{\mathrm{b} 0}=0 \quad \text { on } \partial K \text {. }
\end{aligned}
$$

and

$$
\begin{aligned}
& \epsilon \nabla^{2} V_{\mathrm{b}}^{\epsilon}+\tilde{a} \cdot \nabla B_{\mathrm{b}}^{\epsilon}=-\epsilon \text { in } K, \\
& \epsilon \nabla^{2} B_{\mathrm{b}}^{\epsilon}+\tilde{a} \cdot \nabla V_{\mathrm{b}}^{\epsilon}=0 \quad \text { in } K, \\
& V_{\mathrm{b}}^{\epsilon}=B_{\mathrm{b}}^{\epsilon}=0 \quad \text { on } \partial K .
\end{aligned}
$$

As the components $V_{\mathrm{b}}^{\epsilon}$ and $B_{\mathrm{b}}^{\epsilon}$ appears on the right-hand side of (9), they are simply related with consistency of the method and plays no role in the stability. Therefore it is enough to prove that the form $c_{h}$ is coercive over the reduced space $\mathscr{V}_{h 0} \times \mathscr{B}_{h 0} \times \mathscr{V}_{1} \times \mathscr{B}_{1}$ where

$$
\begin{aligned}
& \mathscr{V}_{h 0}=\left\{V_{h 0}=V_{1}+V_{\mathrm{b} 0}: V_{1} \in \mathscr{V}_{1}\right\}, \\
& \mathscr{B}_{h 0}=\left\{B_{h 0}=B_{1}+B_{\mathrm{b} 0}: B_{1} \in \mathscr{B}_{1}\right\} .
\end{aligned}
$$

We note that the bubble components of $V_{h 0}$ or $B_{h 0}$ are completely determined by their linear counterparts through Eq. (19). We further define our new variables $U_{\mathrm{b} 0}^{1}$ and $U_{\mathrm{b} 0}^{2}$ by $U_{\mathrm{b} 0}^{1}=V_{\mathrm{b} 0}+B_{\mathrm{b} 0}$ and $U_{\mathrm{b} 0}^{2}=V_{\mathrm{b} 0}-B_{\mathrm{b} 0}$. The functions $U_{\mathrm{b} 0}^{1}$ and $U_{\mathrm{b} 0}^{2}$ obviously satisfies the following system of equations

$$
\begin{aligned}
& \epsilon \nabla^{2} U_{\mathrm{b} 0}^{1}+\tilde{a} \cdot \nabla U_{\mathrm{b} 0}^{1}=-\tilde{a} \cdot \nabla B_{1}-\tilde{a} \cdot \nabla V_{1} \text { in } K, \\
& \epsilon \nabla^{2} U_{\mathrm{b} 0}^{2}-\tilde{a} \cdot \nabla U_{\mathrm{b} 0}^{2}=-\tilde{a} \cdot \nabla B_{1}+\tilde{a} \cdot \nabla V_{1} \quad \text { in } K, \\
& U_{\mathrm{b} 0}^{1}=U_{\mathrm{b} 0}^{2}=0 \quad \text { on } \partial K
\end{aligned}
$$

and their asymptotical solutions $\widehat{U}_{\mathrm{b} 0}^{1}$ and $\widehat{U}_{\mathrm{b} 0}^{2}$ satisfies

$$
\begin{aligned}
& \tilde{a} \cdot \nabla \widehat{U}_{\mathrm{b} 0}^{1}=-\tilde{a} \cdot \nabla B_{1}-\tilde{a} \cdot \nabla V_{1} \quad \text { in } K, \quad \widehat{U}_{\mathrm{b} 0}^{1}=0 \quad \text { on } \partial K_{2}, \\
& -\tilde{a} \cdot \nabla \widehat{U}_{\mathrm{b} 0}^{2}=-\tilde{a} \cdot \nabla B_{1}+\tilde{a} \cdot \nabla V_{1} \quad \text { in } K, \quad \widehat{U}_{\mathrm{b} 0}^{2}=0 \quad \text { on } \partial K_{1} .
\end{aligned}
$$

Let us consider the form $c_{h}$ over the reduced space. Using integration by parts and (15) we have

$$
\begin{aligned}
c_{h}\left(V_{\mathrm{b} 0}, B_{\mathrm{b} 0} ;-V_{1},-B_{1}\right)= & \sum_{K}\left(\left(B_{\mathrm{b} 0}, \tilde{a} \cdot \nabla V_{1}\right)_{K}+\left(V_{\mathrm{b} 0}, \tilde{a} \cdot \nabla B_{1}\right)_{K}\right) \\
= & \sum_{K}\left(\left(\widehat{B}_{\mathrm{b} 0}, \tilde{a} \cdot \nabla V_{1}\right)_{K}+\left(B_{\mathrm{b} 0}-\widehat{B}_{\mathrm{b} 0}, \tilde{a} \cdot \nabla V_{1}\right)_{K}\right. \\
& \left.+\left(\widehat{V}_{\mathrm{b} 0}, \tilde{a} \cdot \nabla B_{1}\right)_{K}+\left(V_{\mathrm{b} 0}-\widehat{V}_{\mathrm{b} 0}, \tilde{a} \cdot \nabla B_{1}\right)_{K}\right) \\
= & \sum_{K}\left(\frac{2 h_{a}}{3|\tilde{a}|}\left\|\tilde{a} \cdot \nabla V_{1}\right\|_{K}^{2}+\frac{2 h_{a}}{3|\tilde{a}|}\left\|\tilde{a} \cdot \nabla B_{1}\right\|_{K}^{2}\right. \\
& \left.+\left(B_{\mathrm{b} 0}-\widehat{B}_{\mathrm{b} 0}, \tilde{a} \cdot \nabla V_{1}\right)_{K}+\left(V_{\mathrm{b} 0}-\widehat{V}_{\mathrm{b} 0}, \tilde{a} \cdot \nabla B_{1}\right)_{K}\right) .
\end{aligned}
$$

To obtain a stability estimate, we need to prove that the third and the fourth term in this sum is dominated by the first two terms. This can be achieved by the virtue of Lemma 3 in [12] and Lemma 1 in [1]. Observe that $V_{\mathrm{b} 0}-\widehat{V}_{\mathrm{b} 0}$ and $B_{\mathrm{b} 0}-\widehat{B}_{\mathrm{b} 0}$ can be rewritten in terms of our new variables as follows: 


$$
\begin{aligned}
& V_{\mathrm{b} 0}-\widehat{V}_{\mathrm{b} 0}=\left(U_{\mathrm{b} 0}^{1}-\widehat{U}_{\mathrm{b} 0}^{1}\right)+\left(U_{\mathrm{b} 0}^{2}-\widehat{U}_{\mathrm{b} 0}^{2}\right), \\
& B_{\mathrm{b} 0}-\widehat{B}_{\mathrm{b} 0}=\left(U_{\mathrm{b} 0}^{1}-\widehat{U}_{\mathrm{b} 0}^{1}\right)-\left(U_{\mathrm{b} 0}^{2}-\widehat{U}_{\mathrm{b} 0}^{2}\right) .
\end{aligned}
$$

Define the operators $L_{1}$ and $L_{2}$ as $L_{1}=\epsilon \nabla^{2}+\tilde{a} \cdot \nabla$ and $L_{2}=\epsilon \nabla^{2}-\tilde{a} \cdot \nabla$. We further observe that $U_{\mathrm{b} 0}^{1}-\widehat{U}_{\mathrm{b} 0}^{1}$ and $U_{\mathrm{b} 0}^{2}-\widehat{U}_{\mathrm{b} 0}^{2}$ satisfy the following differential equations:

$$
\begin{aligned}
& L_{1}\left(\widehat{U}_{\mathrm{b} 0}^{1}-U_{\mathrm{b} 0}^{1}\right)=\epsilon \nabla^{2} \widehat{U}_{\mathrm{b} 0}^{1} \quad \text { in } K, \\
& \widehat{U}_{\mathrm{b} 0}^{1}-U_{\mathrm{b} 0}^{1}=0 \quad \text { on } \partial K_{2}, \\
& \widehat{U}_{\mathrm{b} 0}^{1}-U_{\mathrm{b} 0}^{1}=V_{1}\left(x_{Q}\right)+B_{1}\left(x_{Q}\right)-V_{1}\left(x_{P}\right)-B_{1}\left(x_{P}\right) \text { on } \partial K_{1}, \\
& L_{2}\left(\widehat{U}_{\mathrm{b} 0}^{2}-U_{\mathrm{b} 0}^{2}\right)=\epsilon \nabla^{2} \widehat{U}_{\mathrm{b} 0}^{2} \quad \text { in } K, \\
& \widehat{U}_{\mathrm{b} 0}^{2}-U_{\mathrm{b} 0}^{2}=0 \quad \text { on } \partial K_{1} \\
& \widehat{U}_{\mathrm{b} 0}^{2}-U_{\mathrm{b} 0}^{2}=B_{1}\left(x_{Q}\right)-V_{1}\left(x_{Q}\right)-B_{1}\left(x_{P}\right)+V_{1}\left(x_{P}\right) \text { on } \partial K_{2} .
\end{aligned}
$$

Now we can apply Lemma 3 from [12] and Lemma 1 from [1] to the differences $U_{\mathrm{b} 0}^{1}-\widehat{U}_{\mathrm{b} 0}^{1}$ and $U_{\mathrm{b} 0}^{2}-\widehat{U}_{\mathrm{b} 0}^{2}$ to get the bounds

$$
\begin{aligned}
& \left\|\widehat{U}_{\mathrm{b} 0}^{1}-U_{\mathrm{b} 0}^{1}\right\|_{K} \leqslant C C^{\prime}\left\|\tilde{a} \cdot \nabla V_{1}+\tilde{a} \cdot \nabla B_{1}\right\|_{K}, \\
& \left\|\widehat{U}_{\mathrm{b} 0}^{2}-U_{\mathrm{b} 0}^{2}\right\|_{K} \leqslant C C^{\prime}\left\|\tilde{a} \cdot \nabla V_{1}-\tilde{a} \cdot \nabla B_{1}\right\|_{K},
\end{aligned}
$$

where the constant $C^{\prime}$ is given by

$$
C^{\prime}=C^{\prime}\left(\epsilon, h_{a},|\tilde{a}|\right)=\left(\frac{\epsilon^{1 / 2} h_{a}^{1 / 2}}{|\tilde{a}|^{3 / 2}}+\frac{\epsilon}{|\tilde{a}|^{2}}\right) .
$$

We remark that these two lemmas holds only if no edge in the triangulation is aligned with the direction of the flow. The relation (23) and the application of the triangle inequality enable us to write

$$
\begin{aligned}
& \left\|V_{\mathrm{b} 0}-\widehat{V}_{\mathrm{b} 0}\right\|_{K} \leqslant 2 C C^{\prime}\left(\left\|\tilde{a} \cdot \nabla V_{1}\right\|_{K}+\left\|\tilde{a} \cdot \nabla B_{1}\right\|_{K}\right), \\
& \left\|B_{\mathrm{b} 0}-\widehat{B}_{\mathrm{b} 0}\right\|_{K} \leqslant 2 C C^{\prime}\left(\left\|\tilde{a} \cdot \nabla V_{1}\right\|_{K}+\left\|\tilde{a} \cdot \nabla B_{1}\right\|_{K}\right) .
\end{aligned}
$$

Let us consider the last two terms in (22). By Cauchy-Schwartz inequality

$$
\begin{aligned}
& \left|\left(V_{\mathrm{b} 0}-\widehat{V}_{\mathrm{b} 0}, \tilde{a} \cdot \nabla B_{1}\right)\right| \leqslant 2 C C^{\prime}\left(\left\|\tilde{a} \cdot \nabla V_{1}\right\|_{K}\left\|\tilde{a} \cdot \nabla B_{1}\right\|_{K}+\left\|\tilde{a} \cdot \nabla B_{1}\right\|_{K}^{2}\right), \\
& \left|\left(B_{\mathrm{b} 0}-\widehat{B}_{\mathrm{b} 0}, \tilde{a} \cdot \nabla V_{1}\right)\right| \leqslant 2 C C^{\prime}\left(\left\|\tilde{a} \cdot \nabla V_{1}\right\|_{K}^{2}+\left\|\tilde{a} \cdot \nabla V_{1}\right\|_{K}\left\|\tilde{a} \cdot \nabla B_{1}\right\|_{K}\right) .
\end{aligned}
$$

We employ the well-known inequality $-a b \geqslant-\frac{a^{2}}{2}-\frac{b^{2}}{2}$ to get the desired result. Starting from (22) we have, 


$$
\begin{aligned}
c_{h}\left(V_{\mathrm{b} 0}, B_{\mathrm{b} 0} ;-V_{1},-B_{1}\right)_{K}= & \sum_{K}\left(\frac{2}{3} \frac{h_{a}}{|\tilde{a}|}\left(\left\|\tilde{a} \cdot \nabla V_{1}\right\|_{K}^{2}+\left\|\tilde{a} \cdot \nabla B_{1}\right\|_{K}^{2}\right)\right. \\
& \left.+\left(B_{\mathrm{b} 0}-\widehat{B}_{\mathrm{b} 0}, \tilde{a} \cdot \nabla V_{1}\right)_{K}+\left(V_{\mathrm{b} 0}-\widehat{V}_{\mathrm{b} 0}, \tilde{a} \cdot \nabla B_{1}\right)_{K}\right) \\
\geqslant & \sum_{K}\left(\frac{2}{3} \frac{h_{a}}{|\tilde{a}|}\left(\left\|\tilde{a} \cdot \nabla V_{1}\right\|_{K}^{2}+\left\|\tilde{a} \cdot \nabla B_{1}\right\|_{K}^{2}\right)\right. \\
& \left.-2 C C^{\prime}\left(\left\|\tilde{a} \cdot \nabla V_{1}\right\|_{K}^{2}+2\left\|\tilde{a} \cdot \nabla V_{1}\right\|_{K}\left\|\tilde{a} \cdot \nabla B_{1}\right\|_{K}+\left\|\tilde{a} \cdot \nabla B_{1}\right\|_{K}^{2}\right)\right) \\
\geqslant & \sum_{K}\left(\frac{2}{3} \frac{h_{a}}{|\tilde{a}|}\left(\left\|\tilde{a} \cdot \nabla V_{1}\right\|_{K}^{2}+\left\|\tilde{a} \cdot \nabla B_{1}\right\|_{K}^{2}\right)\right. \\
& \left.-4 C C^{\prime}\left\|\tilde{a} \cdot \nabla V_{1}\right\|_{K}^{2}-4 C C^{\prime}\left\|\tilde{a} \cdot \nabla B_{1}\right\|_{K}^{2}\right) \\
\geqslant & \sum_{K}\left(\frac{2}{3} \frac{h_{a}}{|\tilde{a}|}\left\|\tilde{a} \cdot \nabla V_{1}\right\|_{K}^{2}-4 C\left(\frac{\epsilon^{1 / 2} h_{a}^{1 / 2}}{|\tilde{a}|^{3 / 2}}+\frac{\epsilon}{|\tilde{a}|^{2}}\right)\left\|\tilde{a} \cdot \nabla V_{1}\right\|_{K}^{2}\right. \\
& \left.+\frac{2}{3} \frac{h_{a}}{|\tilde{a}|}\left\|\tilde{a} \cdot \nabla B_{1}\right\|_{K}^{2}-4 C\left(\frac{\epsilon^{1 / 2} h_{a}^{1 / 2}}{|\tilde{a}|^{3 / 2}}+\frac{\epsilon}{|\tilde{a}|^{2}}\right)\left\|\tilde{a} \cdot \nabla B_{1}\right\|_{K}^{2}\right) \\
\geqslant & \sum_{K}\left(\frac{1}{6} \frac{h_{a}}{|\tilde{a}|}\left\|\tilde{a} \cdot \nabla V_{1}\right\|_{K}^{2}+\frac{1}{6} \frac{h_{a}}{|\tilde{a}|}\left\|\tilde{a} \cdot \nabla B_{1}\right\|_{K}^{2}\right)
\end{aligned}
$$

where we have assumed $\epsilon \leqslant h_{a}|\tilde{a}| \min \left\{1, \frac{1}{256 C^{2}}\right\}$ for the last inequality and considered the cases $1 \leqslant 256 C^{2}$ and $1>256 C^{2}$ separately. We state this result in the following theorem.

Theorem 1. Let $V_{\mathrm{b} 0}$ and $B_{\mathrm{b} 0}$ be defined as in (19). Suppose that no edge in the triangulation is aligned with the direction of the flow and that

$$
\epsilon \leqslant h_{a}|\tilde{a}| \min \left\{1, \frac{1}{256 C^{2}}\right\}
$$

for all triangles $K$ in the triangulation. Then

$$
c_{h}\left(V_{\mathrm{b} 0}, B_{\mathrm{b} 0} ;-V_{1},-B_{1}\right) \geqslant \sum_{K}\left(\frac{1}{6} \frac{h_{a}}{|\tilde{a}|}\left\|\tilde{a} \cdot \nabla V_{1}\right\|_{K}^{2}+\frac{1}{6} \frac{h_{a}}{|\tilde{a}|}\left\|\tilde{a} \cdot \nabla B_{1}\right\|_{K}^{2}\right) .
$$

\section{Numerical results}

In this section we report three series of experiments for the MHD flow problem using the numerical method (9) introduced in Section 3. To employ the numerical method we have to find the residual-free bubble part $\left\{V_{\mathrm{b}}, B_{\mathrm{b}}\right\}$ of the numerical solution $\left\{V_{h}, B_{h}\right\}$ and assemble the contribution coming from the bubble part of the solution to the global stiffness matrix. This can be done by a two-level finite element method (TLFEM) as described in [12]. In the TLFEM, we set another layer of mesh - call it submesh - inside each element $K$ and apply a non-standard finite element method to find approximations to the bubble functions $\left\{V_{\mathrm{b}}, B_{\mathrm{b}}\right\}$. We use a $4 \times 4$ uniform submesh in our experiments. However, starting from 1000 , Hartmann number becomes high enough so that we can replace the bubble functions $\left\{V_{\mathrm{b}}, B_{\mathrm{b}}\right\}$ with their asymptotical counterparts $\left\{\widehat{V}_{\mathrm{b}}, \widehat{B}_{\mathrm{b}}\right\}$. Thus we replace the terms containing bubble functions $V_{\mathrm{b}}$ or $B_{\mathrm{b}}$ in the numerical 


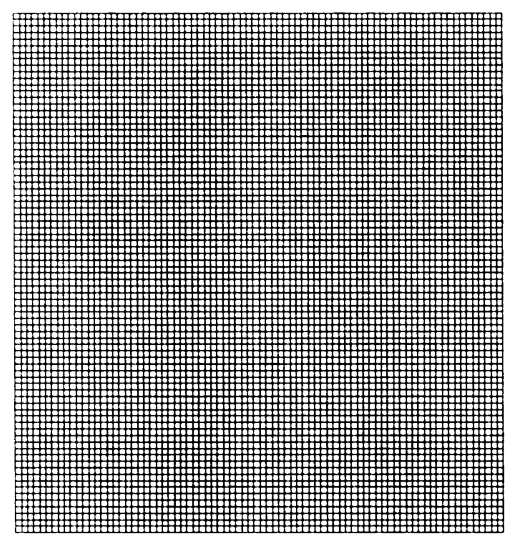

Fig. 3. Mesh employed in test problems.

method (9) with the expressions in (18). We note that this replacement makes implementation more convenient and computation much cheaper.

The cross-section of the duct for the MHD flow is a square defined by $|x| \leqslant 1$ and $|y| \leqslant 1$, taking the origin at the centre of the section. We choose a fix uniform $80 \times 80$ mesh of four-node quadrilateral elements for all test problems (Fig. 3).

In our computations we use a Fortran77 compiler on a Linux platform and all the graphics are obtained by using the visualization software VIGIE 1.7 developed by INRIA.

\subsection{Shercliff's problem: transverse magnetic field on a square channel}

This is an example with analytical solution where the walls of the channel are insulated $(B=0)$. The velocity is zero on the solid walls $(V=0)$. The external magnetic field is applied in the direction of the $x$-axis $(\alpha=\pi / 2)$ (see Fig. 4 for the problem statement). The analytical solution of this problem is given by Shercliff [10] so that we can compare the approximate solution with the exact solution.

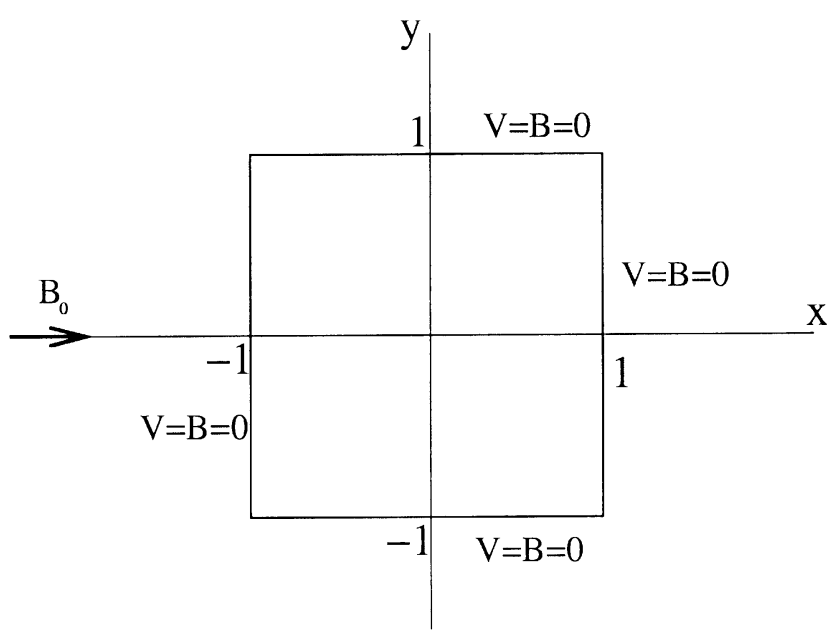

Fig. 4. Shercliff's problem. 
Table 1

The flow field and the magnetic field in Shercliff's problem at $M=100$

\begin{tabular}{|c|c|c|c|c|c|}
\hline$x$ & $y$ & $V_{h}$ & $V_{\text {exact }}$ & $B_{h}$ & $B_{\text {exact }}$ \\
\hline 0.00 & 0.00 & 0.0100000 & 0.0100000 & 0.0000000 & 0.0000000 \\
\hline 0.25 & 0.00 & 0.0100000 & 0.0100000 & -0.0025000 & -0.0025000 \\
\hline 0.50 & 0.00 & 0.0100000 & 0.0100000 & -0.0050000 & -0.0050000 \\
\hline 0.75 & 0.00 & 0.0100000 & 0.0100000 & -0.0075000 & -0.0075000 \\
\hline 0.00 & 0.25 & 0.0100000 & 0.0100000 & 0.0000000 & 0.0000000 \\
\hline 0.25 & 0.25 & 0.0100000 & 0.0100000 & -0.0025000 & -0.0025000 \\
\hline 0.50 & 0.25 & 0.0099999 & 0.0100000 & -0.0050000 & -0.0050000 \\
\hline 0.75 & 0.25 & 0.0099999 & 0.0099999 & -0.0074999 & -0.0074999 \\
\hline 0.00 & 0.50 & 0.0099993 & 0.0099992 & 0.0000000 & -0.0000000 \\
\hline 0.25 & 0.50 & 0.0099983 & 0.0099981 & -0.0024984 & -0.0024982 \\
\hline 0.50 & 0.50 & 0.0099947 & 0.0099944 & -0.0049947 & -0.0049944 \\
\hline 0.75 & 0.50 & 0.0099873 & 0.0099868 & -0.0074873 & -0.0074868 \\
\hline 0.00 & 0.75 & 0.0097662 & 0.0097614 & 0.0000000 & 0.0000000 \\
\hline 0.25 & 0.75 & 0.0097209 & 0.0097163 & -0.0023043 & -0.0023030 \\
\hline 0.50 & 0.75 & 0.0095898 & 0.0095858 & -0.0046050 & -0.0046024 \\
\hline 0.75 & 0.75 & 0.0093899 & 0.0093863 & -0.0068903 & -0.0068869 \\
\hline
\end{tabular}

Table 2

The flow field and the magnetic field in Shercliff's problem at $M=500$

\begin{tabular}{llllrr}
\hline$x$ & $y$ & $V_{h}$ & $V_{\text {exact }}$ & \multicolumn{1}{c}{$B_{h}$} & \multicolumn{1}{c}{$B_{\text {exact }}$} \\
\hline 0.00 & 0.00 & 0.0020000 & 0.0020000 & 0.0000000 & 0.0000000 \\
0.25 & 0.00 & 0.0020000 & 0.0020000 & -0.0005000 & -0.0005000 \\
0.50 & 0.00 & 0.0020000 & 0.0020000 & -0.0010000 & -0.0010000 \\
0.75 & 0.00 & 0.0020000 & 0.0020000 & -0.0015000 & -0.0015000 \\
0.00 & 0.25 & 0.0020000 & 0.0020000 & 0.0000000 & 0.0000000 \\
0.25 & 0.25 & 0.0020000 & 0.0020000 & -0.0005000 & -0.0005000 \\
0.50 & 0.25 & 0.0020000 & 0.0020000 & -0.0010000 & -0.0010000 \\
0.75 & 0.25 & 0.0020000 & 0.0020000 & -0.0015000 & -0.0015000 \\
0.00 & 0.50 & 0.0020000 & 0.0020000 & -0.00000000000000 & -0.0000000 \\
0.25 & 0.50 & 0.0020000 & 0.0020000 & -0.0010000 & -0.0005000 \\
0.50 & 0.50 & 0.0020000 & 0.0020000 & 0.0000000 & -0.0010000 \\
0.75 & 0.50 & 0.0020000 & 0.0020000 & -0.0005000 & -0.0015000 \\
0.00 & 0.75 & 0.0020000 & 0.0020000 & -0.0009998 & 0.0000000 \\
0.25 & 0.75 & 0.0019999 & 0.0019999 & -0.0014994 & -0.0004999 \\
0.50 & 0.75 & 0.0019998 & 0.0019997 & & -0.0009997 \\
0.75 & 0.75 & 0.0019994 & 0.0019992 & & -0.0014992 \\
\hline
\end{tabular}

In Tables 1 and 2 we compare the approximate solution with the exact solution for Hartmann numbers 100 and 500, respectively, at several grid points in the first quadrant of the channel. One can see that two results are comparable.

Velocity and magnetic field contours for $M=100,500,1000$ and 10,000 for this problem $(\alpha=\pi / 2)$ are going to be presented with the contours obtained for other values of angle $\alpha(\alpha=\pi / 4$ and $\alpha=\pi / 3)$ in the second problem for comparison. 


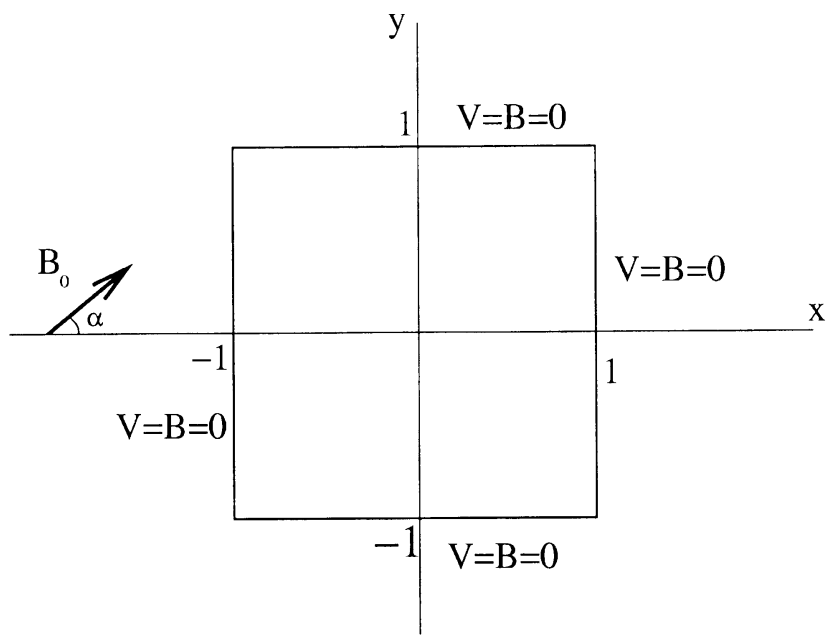

Fig. 5. 2D square-channel problem with externally applied magnetic fields.

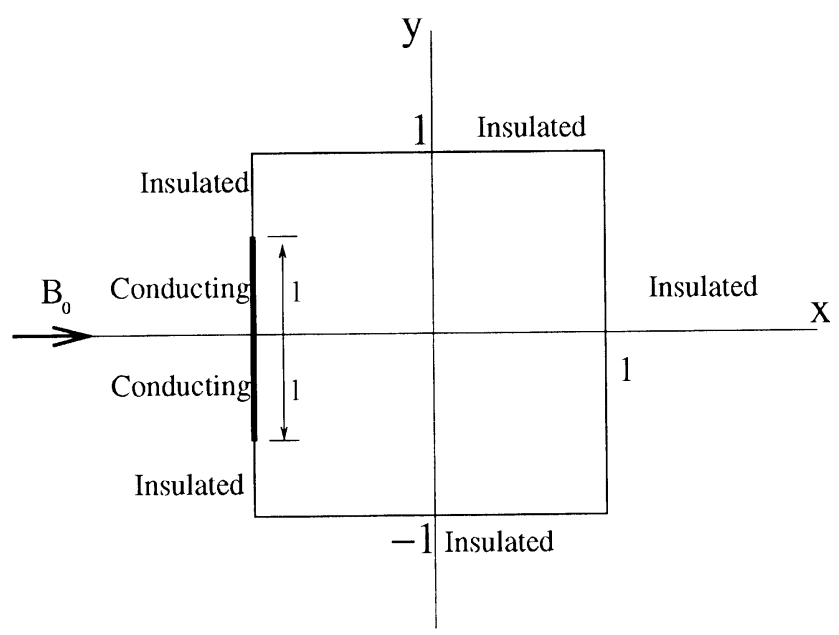

Fig. 6. 2D square-channel problem with conducting boundary.

\section{2. $2 D$ square-channel problem with an oblique applied magnetic fields}

The same MHD problem is considered here by taking externally applied magnetic field making a positive angle with the $x$-axis (see Fig. 5). Computations are carried for values of $\alpha=\pi / 4, \pi / 3$ and $\pi / 2$, respectively. For these values of $\alpha$ velocity contours (or induced magnetic field contours) are given on the same picture. Those graphics are obtained and presented at Hartmann numbers $M=100,500,1000$ and 10,000, respectively, in Figs. 7-14. As Hartmann number increases the boundary layer formation close to the walls for both velocity and induced magnetic field is well observed. Velocity becomes stagnant at the center of the channel. When external magnetic field applies obliquely, the boundary layers are concentrated near the cor- 


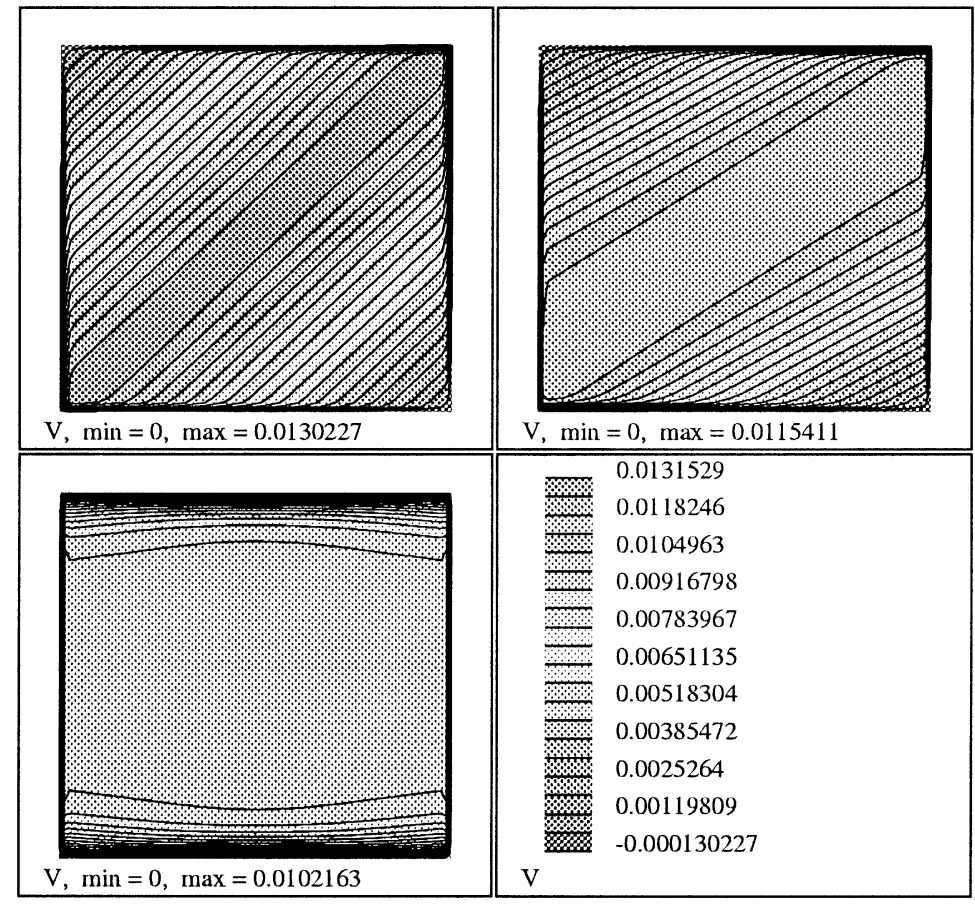

Fig. 7. Velocity field in Problem 5.2 at $M=100(\alpha=\pi / 4, \pi / 3$ and $\pi / 2$, respectively).

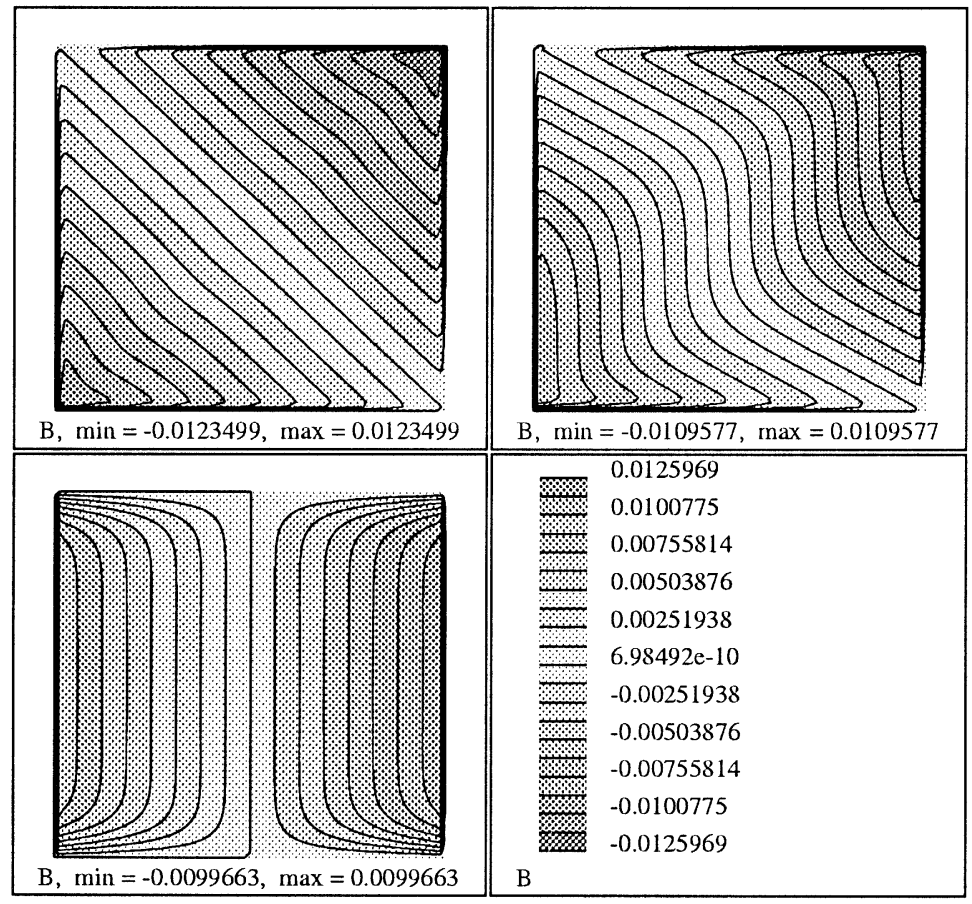

Fig. 8. Magnetic field in Problem 5.2 at $M=100(\alpha=\pi / 4, \pi / 3$ and $\pi / 2$, respectively). 


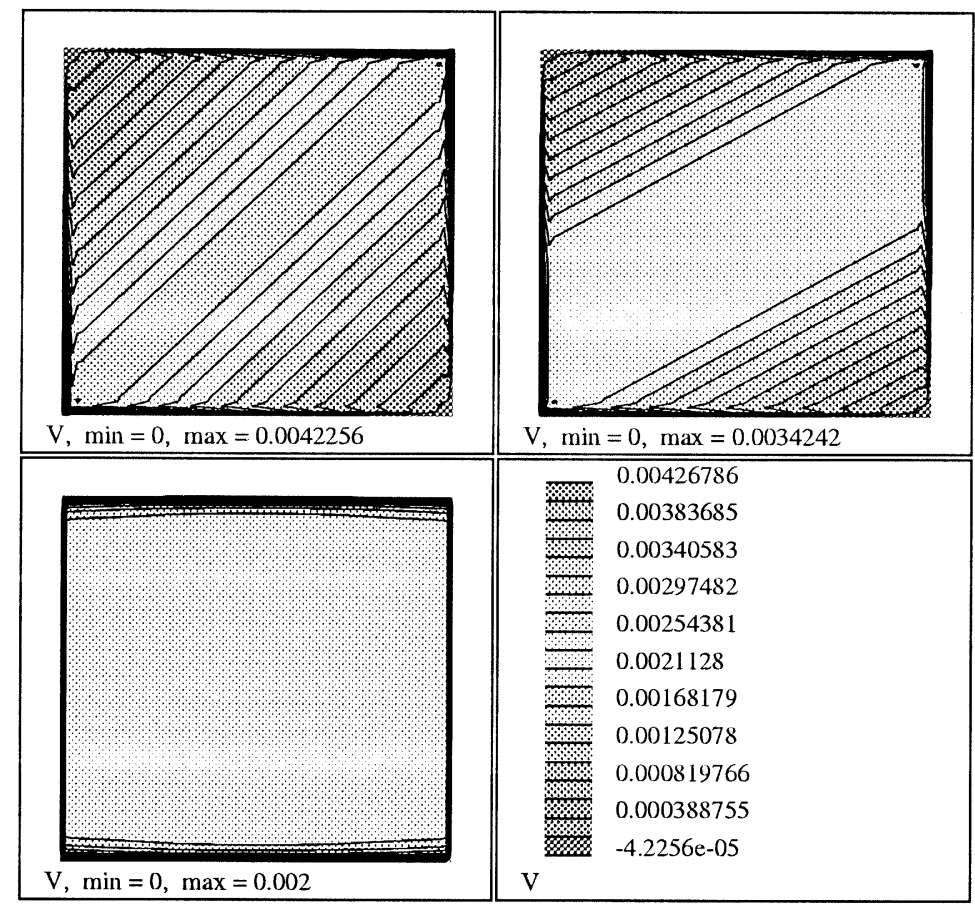

Fig. 9. Velocity field in Problem 5.2 at $M=500(\alpha=\pi / 4, \pi / 3$ and $\pi / 2$, respectively).

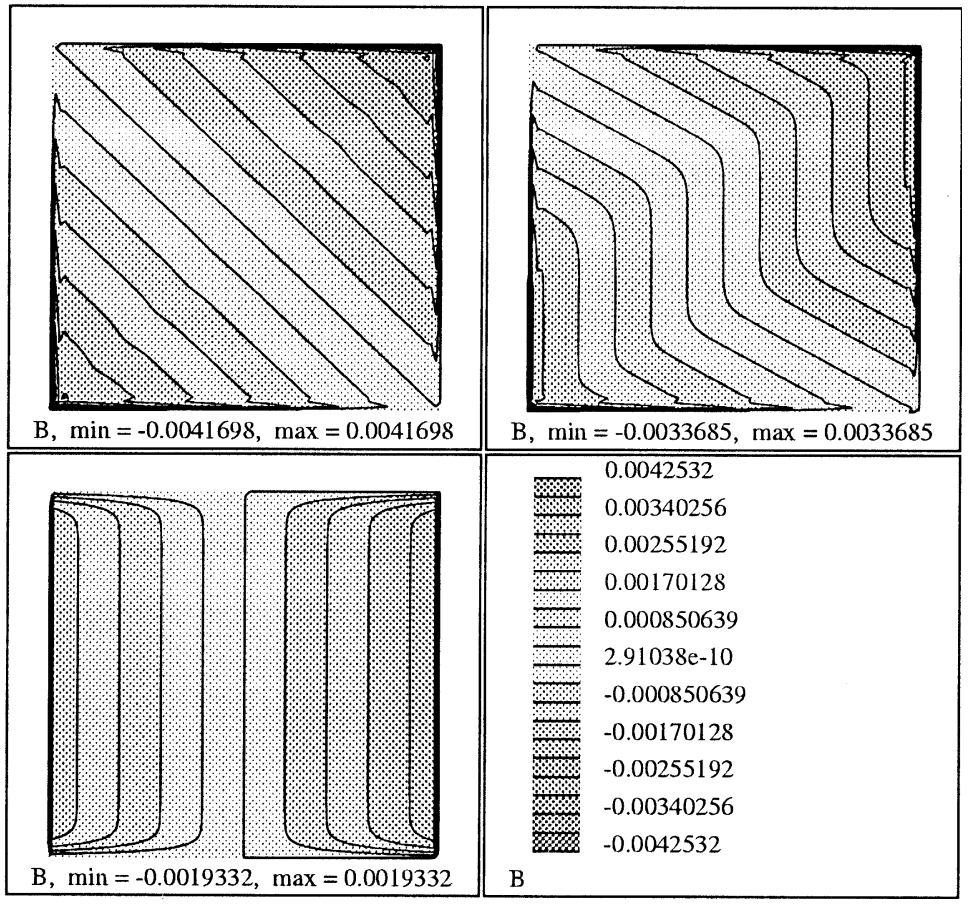

Fig. 10. Magnetic field in Problem 5.2 at $M=500(\alpha=\pi / 4, \pi / 3$ and $\pi / 2$, respectively). 


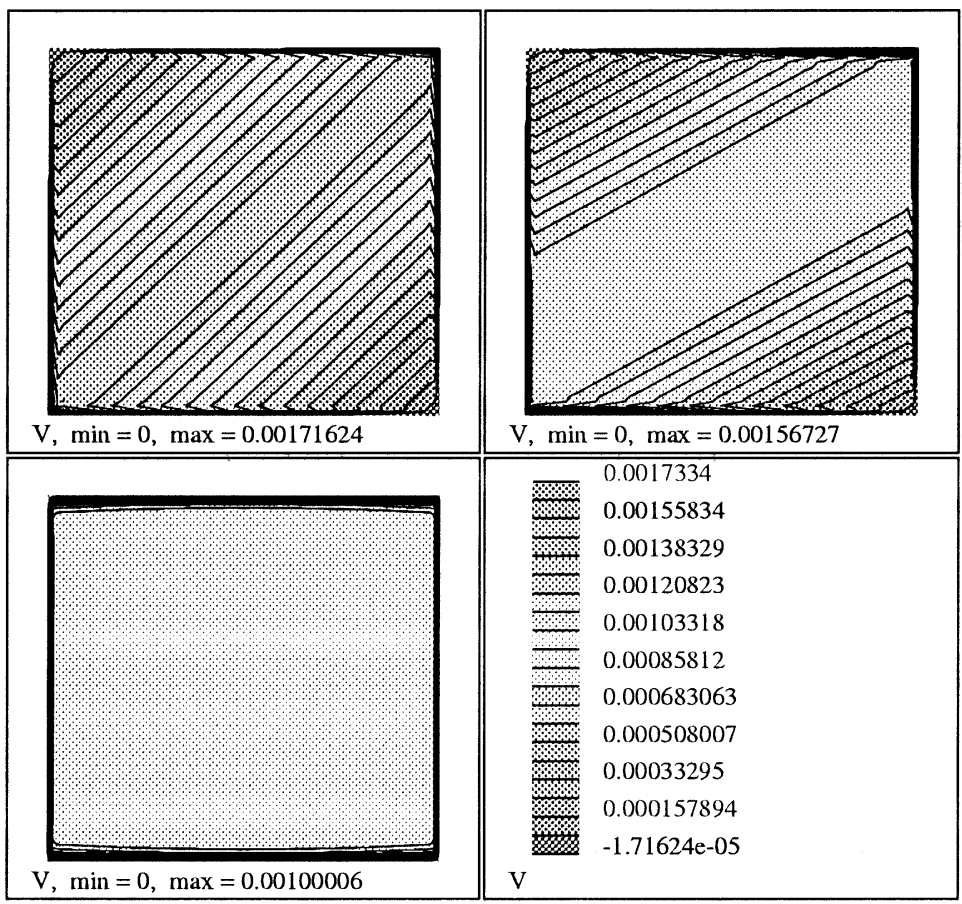

Fig. 11. Velocity field in Problem 5.2 at $M=1000(\alpha=\pi / 4, \pi / 3$ and $\pi / 2$, respectively).

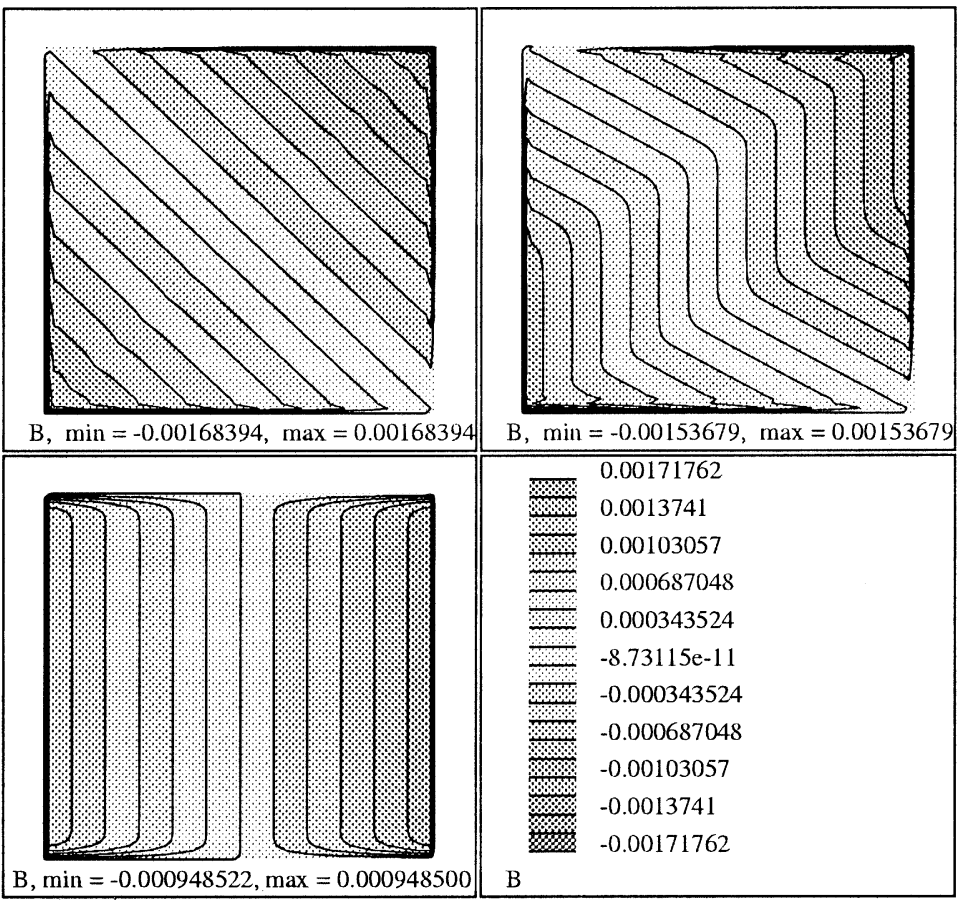

Fig. 12. Magnetic field in Problem 5.2 at $M=1000(\alpha=\pi / 4, \pi / 3$ and $\pi / 2$, respectively). 


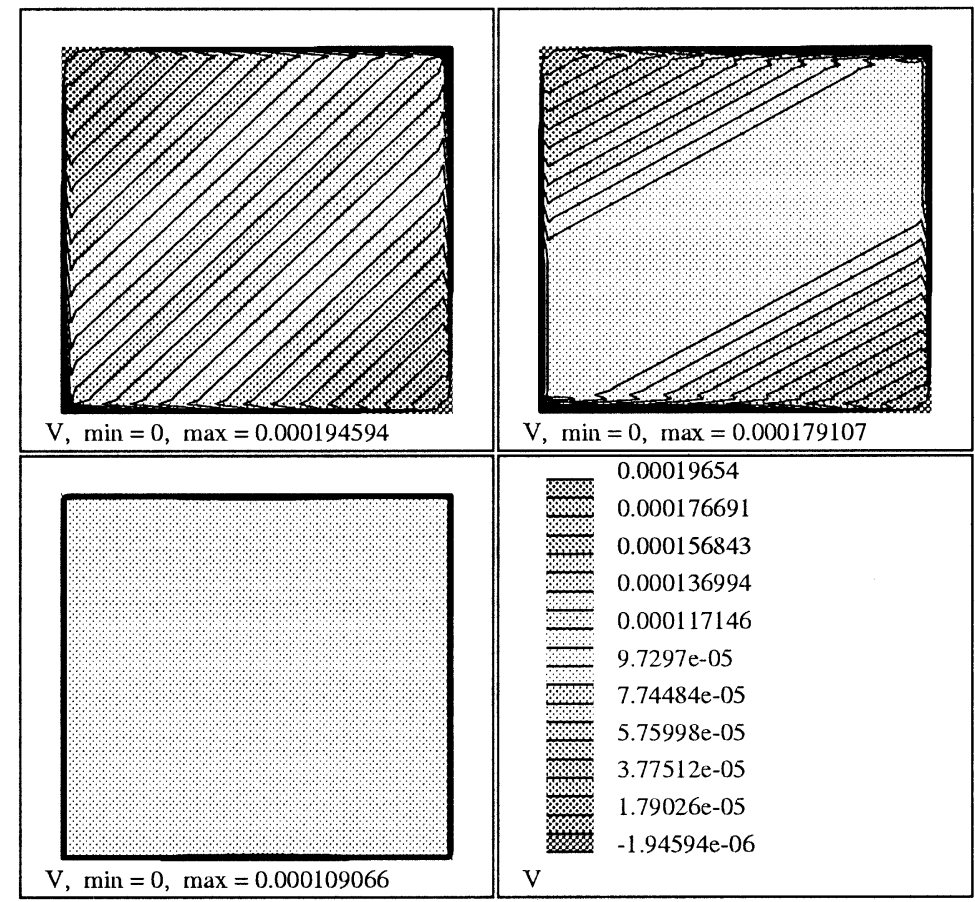

Fig. 13. Velocity field in Problem 5.2 at $M=10,000(\alpha=\pi / 4, \pi / 3$ and $\pi / 2$, respectively).

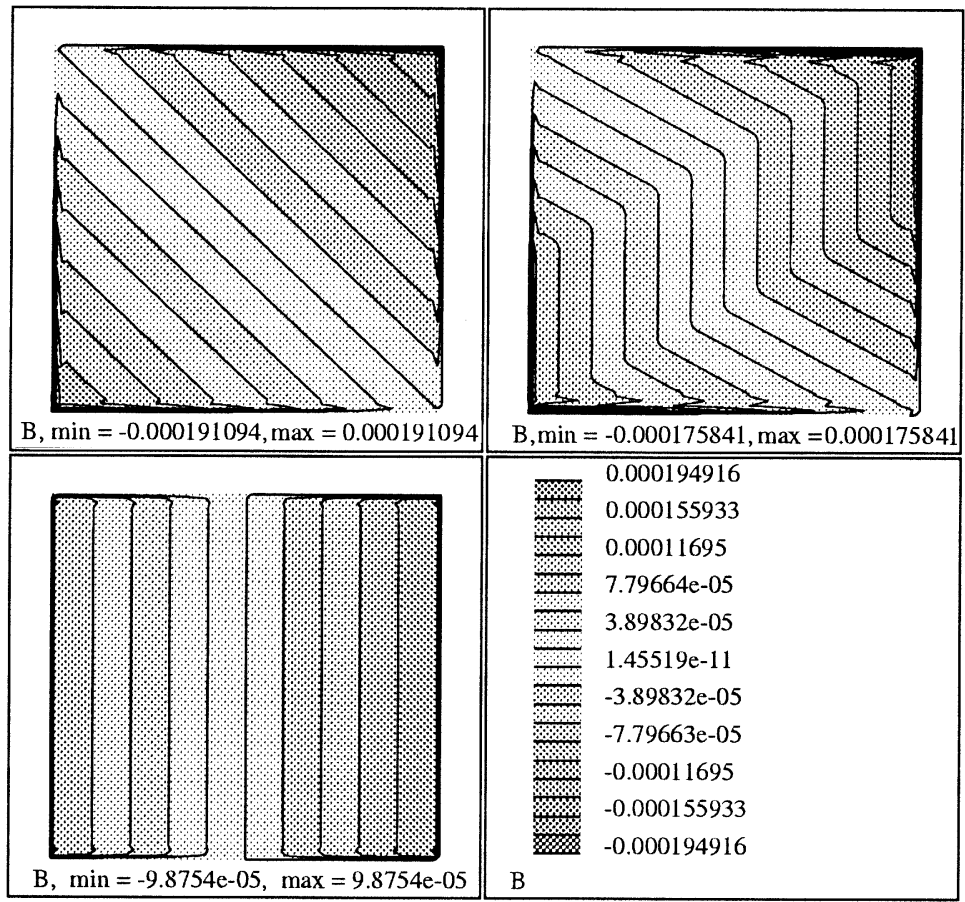

Fig. 14. Magnetic field in Problem 5.2 at $M=10,000(\alpha=\pi / 4, \pi / 3$ and $\pi / 2$, respectively). 
ners in the direction of the field for both solutions of $V$ and $B$. These are the well-known behaviors of the magnetohydrodynamic flow.

\subsection{D square-channel problem with partly conducting boundary}

In this problem external magnetic field $B_{0}$ is perpendicular to the wall at $x=-1$. This wall is electrically insulated except for a length $2 l$ at the center (Fig. 6).

In Figs. 15-24 the velocity and the induced magnetic field contours are presented for several values of $l$ $(l=0.2,0.5$ and 0.7$)$ for the same Hartmann number on the same picture. The values of Hartmann number we test are $M=100,500,1000,10,000$ and 100,000, respectively. As Hartmann number $M$ increases, boundary layers are formed not only close to the walls but also inside of the channel. In front of the discontinuity points $y=-l$ and $y=+l$ parabolic boundary layers are formed in the direction of applied field leading three stagnant regions for the velocity. This behavior can be observed from the figures. For very high values of Hartmann number we note that the numerical solutions show the dominant characteristics of the MHD flow very clearly: Flow in the channel is completely separated into two rectangular flow regions with the boundary layers leaving the center regions stagnant. Thus our FEM scheme with the residual-free bubble functions enables to obtain results of the MHD problem for very high Hartmann numbers.

\section{Conclusions}

We considered the application of the residual-free bubble method to MHD flow problem in a straight channel of uniform cross-section. We showed that the method is stable at moderate and high values of

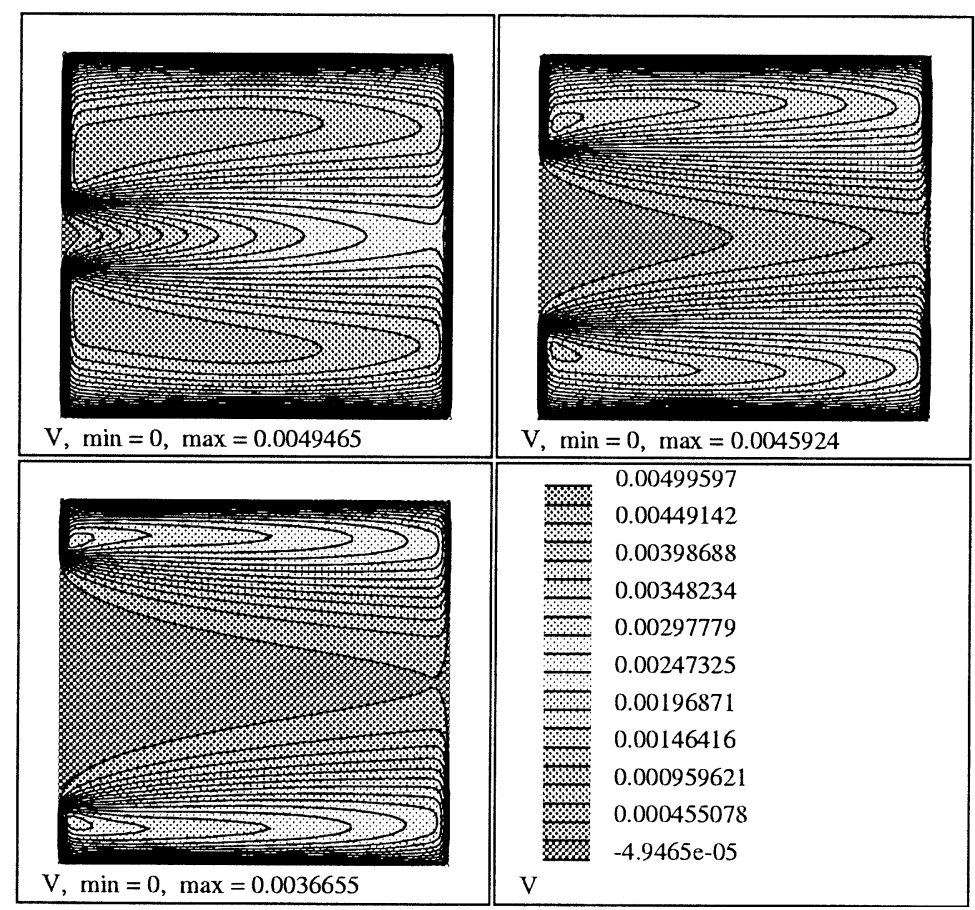

Fig. 15. Velocity field in Problem 5.3 at $M=100(l=0.2,0.5$ and 0.7 , respectively). 


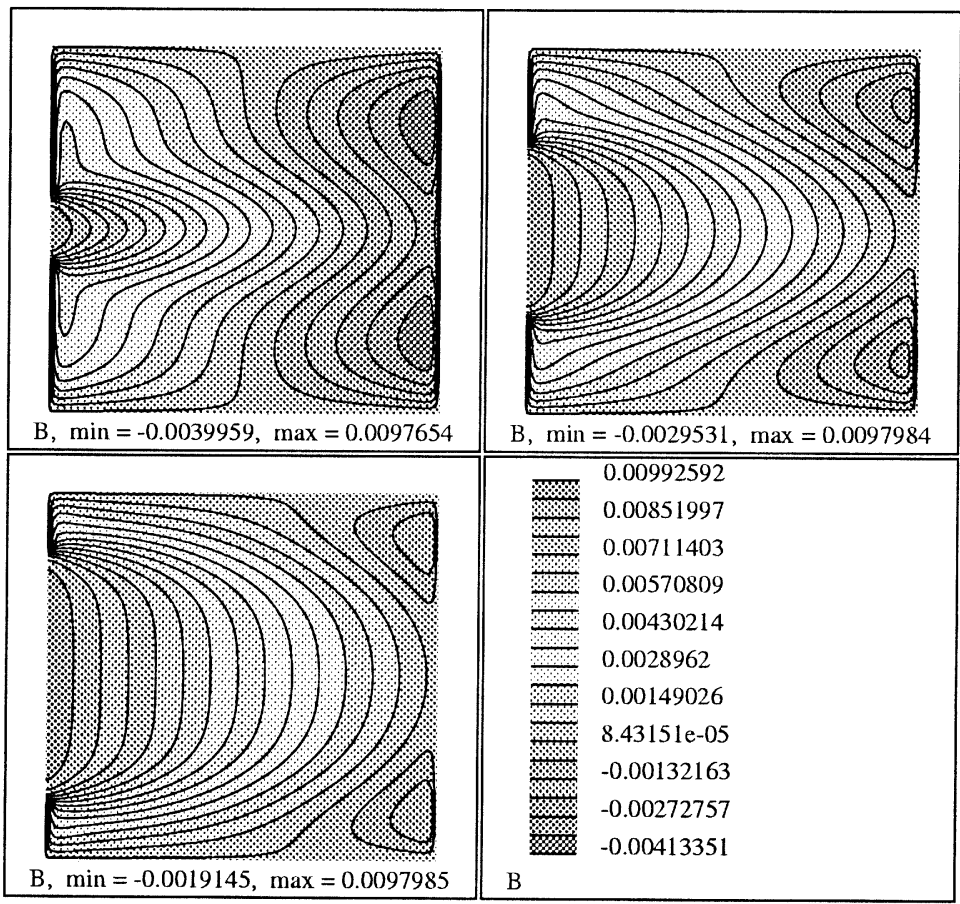

Fig. 16. Magnetic field in Problem 5.3 at $M=100$ ( $l=0.2,0.5$ and 0.7 , respectively).

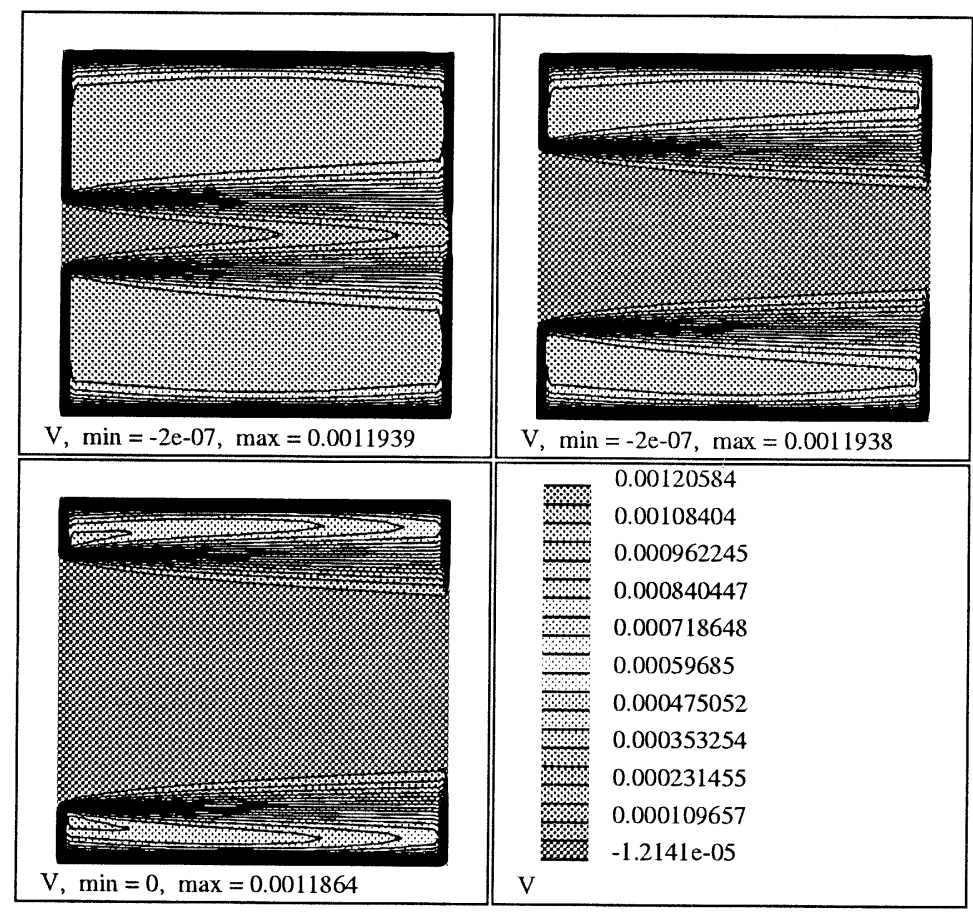

Fig. 17. Velocity field in Problem 5.3 at $M=500(l=0.2,0.5$ and 0.7 , respectively). 


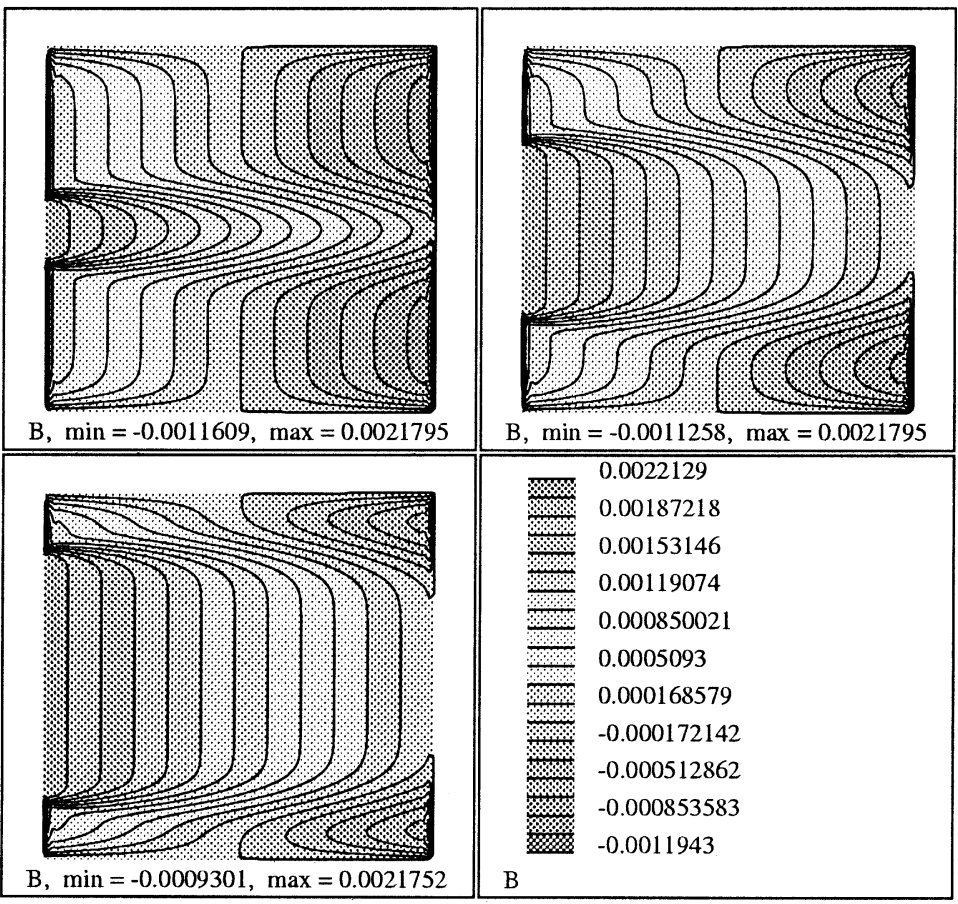

Fig. 18. Magnetic field in Problem 5.3 at $M=500$ ( $l=0.2,0.5$ and 0.7 , respectively).

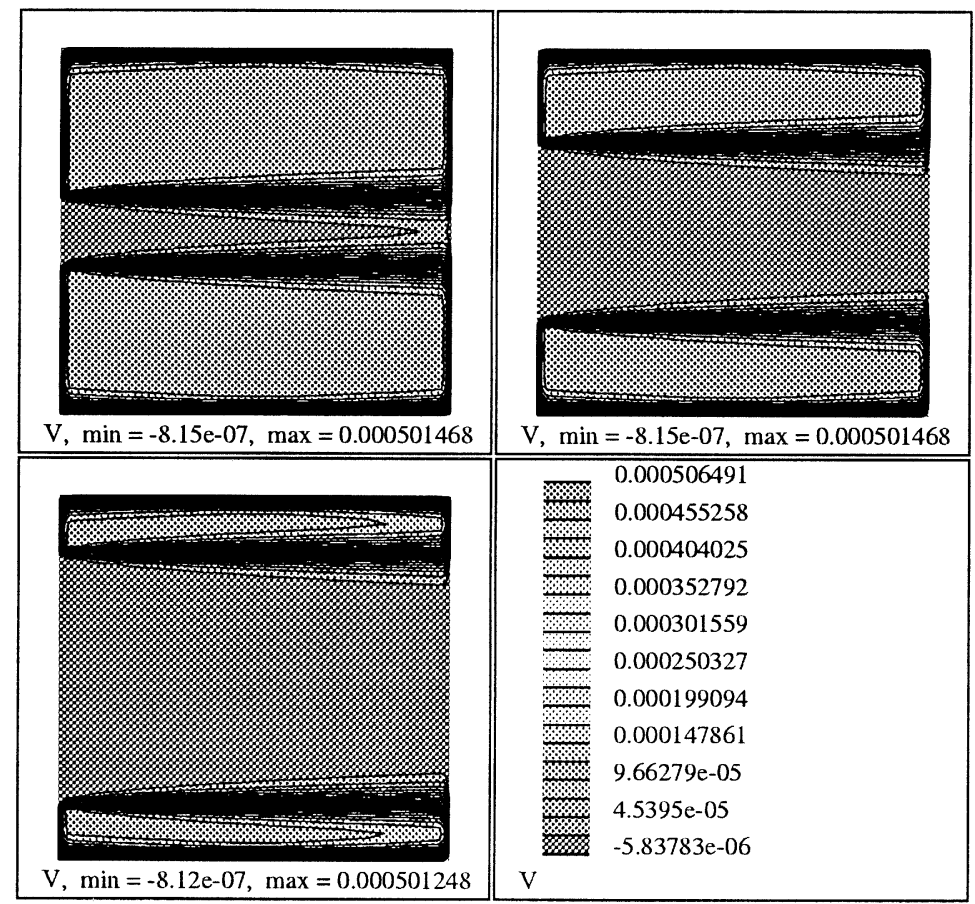

Fig. 19. Velocity field in Problem 5.3 at $M=1000$ ( $l=0.2,0.5$ and 0.7 , respectively). 


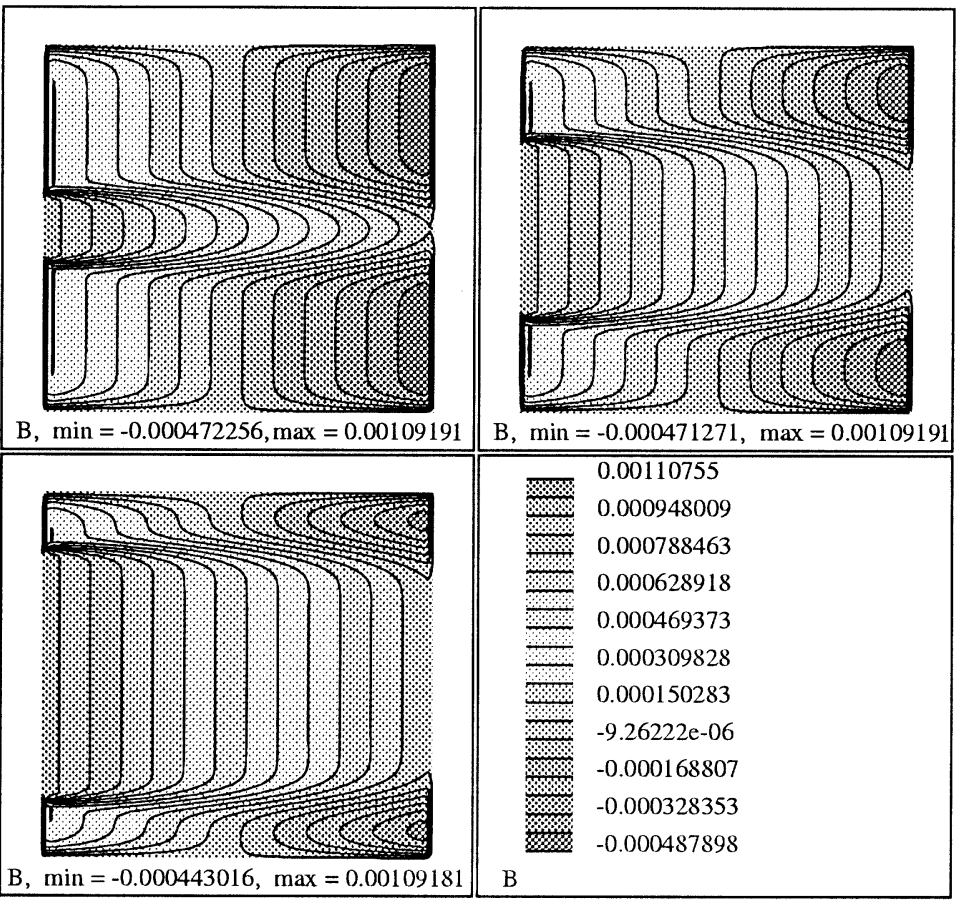

Fig. 20. Magnetic field in Problem 5.3 at $M=1000$ ( $l=0.2,0.5$ and 0.7 , respectively).

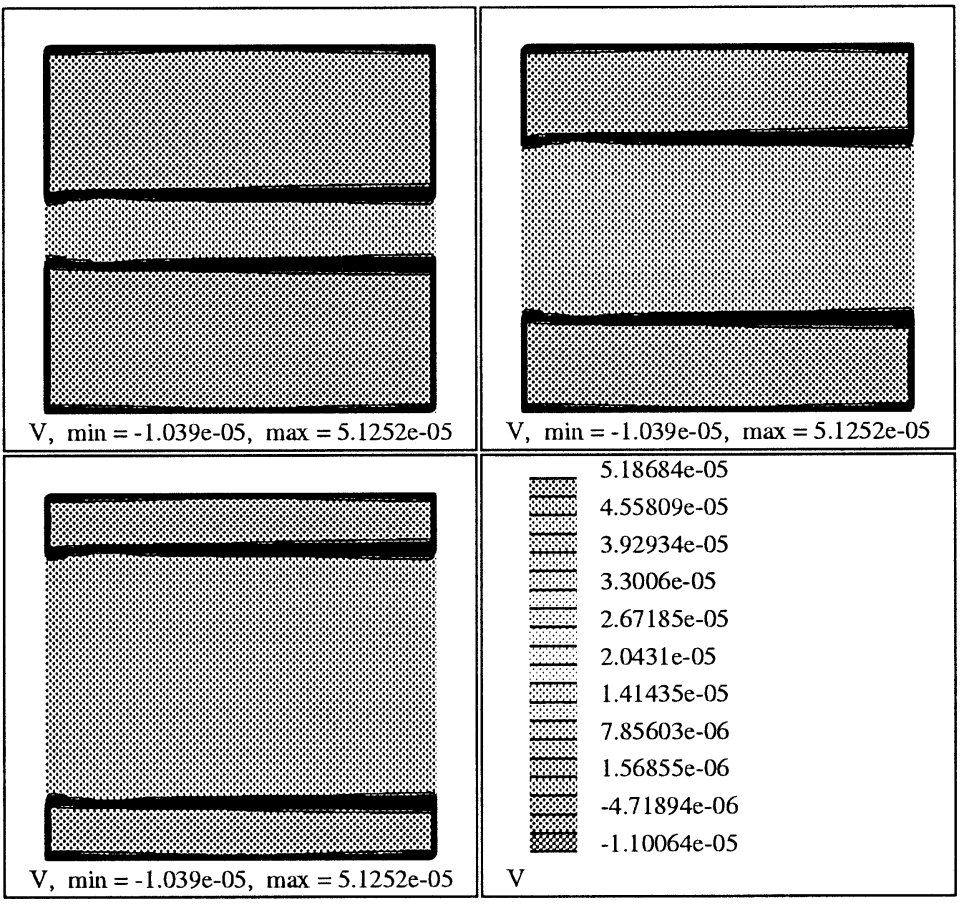

Fig. 21. Velocity field in Problem 5.3 at $M=10,000(l=0.2,0.5$ and 0.7 , respectively). 


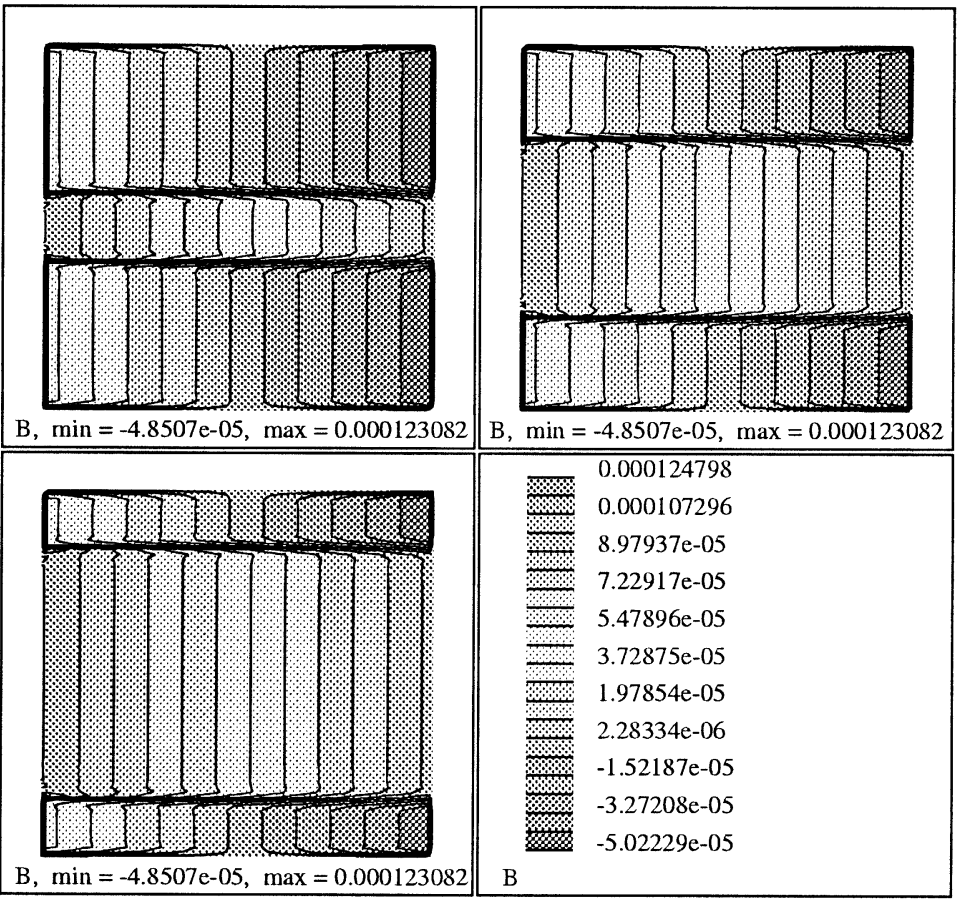

Fig. 22. Magnetic field in Problem 5.3 at $M=10,000(l=0.2,0.5$ and 0.7 , respectively).

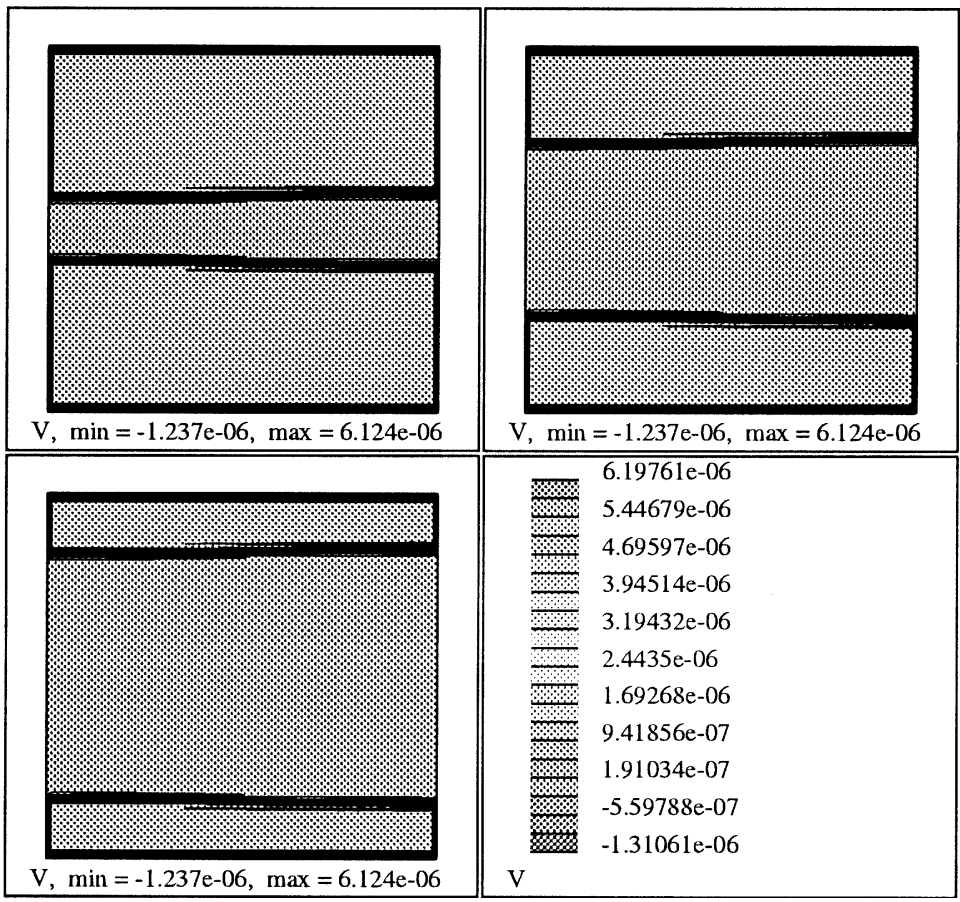

Fig. 23. Velocity field in Problem 5.3 at $M=100,000(l=0.2,0.5$ and 0.7 , respectively). 


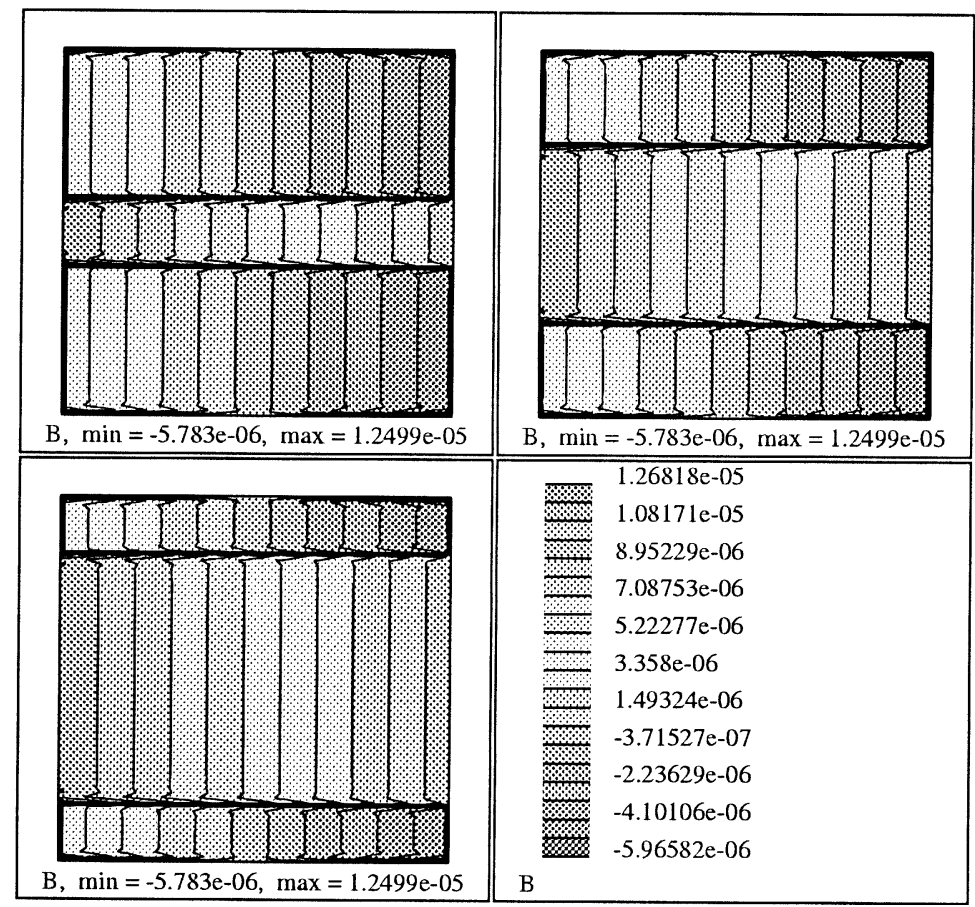

Fig. 24. Magnetic field in Problem 5.3 at $M=100,000$ ( $l=0.2,0.5$ and 0.7 , respectively).

Hartmann number when a partition of triangular elements is employed. Our analysis also led us to some computational trick that make the implementation more convenient. On the basis our of computer experiments we can conclude that the RFB method gives more stable and accurate results than previous numerical method used to solve the MHD problem. We finally note that the present method can be generalized to channels with arbitrary cross-sections.

\section{References}

[1] A. Nesliturk, I. Harari, The nearly-optimal Petrov-Galerkin method for convection-diffusion problems, Comput. Methods Appl. Mech. Engrg. 192 (2003) 2501-2519.

[2] F. Brezzi, L.P. Franca, A. Russo, Further considerations on residual-free bubbles for advective-diffusive equations, Comput. Methods Appl. Mech. Engrg. 166 (1998) 25-33.

[3] F. Brezzi, T.J.R. Hughes, D. Marini, A. Russo, E. Suli, A priori error analysis of a finite element method with residual-free bubbles for advection dominated equations, SIAM J. Numer. Anal. 36 (1999) 1933-1948.

[4] F. Brezzi, A. Russo, Choosing bubbles for advection-diffusion problems, Math. Models Meth. Appl. Sci. 4 (1994) $571-587$.

[5] B. Singh, J. Lal, MHD axial flow in a triangular pipe under transverse magnetic field, Ind. J. Pure Appl. Math. 9 (1978) $101-115$.

[6] B. Singh, J. Lal, MHD axial flow in a triangular pipe under transverse magnetic field parallel to a side of the triangle, Ind. J. Tech. 17 (1979) 184-189.

[7] B. Singh, J. Lal, FEM in MHD channel flow problems, Int. J. Numer. Methods Engrg. 18 (1982) 1104-1111.

[8] B. Singh, J. Lal, FEM for unsteady MHD flow through pipes with arbitrary wall conductivity, Int. J. Numer. Methods Fluids 4 (1984) 291-302.

[9] L.P. Franca, L. Tobiska, Stability of the residual free bubble method for bilinear finite elements on rectangular grids, IMA J. Numer. Anal. 22 (2002) 73-87. 
[10] J.A. Shercliff, Steady motion of conducting fluids in pipes under transverse magnetic fields, Proc. Camb. Philos. Soc. 49 (1953) 136-144.

[11] L. Dragos, Magnetofluid Dynamics, Abacus Press, 1975.

[12] L.P. Pranca, A. Nesliturk, M. Stynes, On the stability of residual-free bubbles for convection-diffusion problems and their approximation by a two-level finite element method, Comput. Methods Appl. Mech. Engrg. 166 (1998) 35-49.

[13] A. Russo, Bubble stabilization of finite element methods for the linearized incompressible Navier-Stokes equations, Comput. Methods Appl. Mech. Engrg. 132 (1996) 335-343.

[14] B. Singh, J. Lal, FEM for MHD channel flow with arbitrary wall conductivity, J. Math. Phys. Sci. 18 (1984) $501-516$.

[15] M. Tezer, Solution of MHD flow in a rectangular duct by differential quadrature method, Comput. Fluids 33 (2004) $533-547$.

[16] M. Tezer-Sezgin, BEM solution of MHD flow in a rectangular duct, Int. J. Numer. Methods Fluids 18 (1994) 937-952.

[17] M. Tezer-Sezgin, S. Koksal, FEM for solving MHD flow in a rectangular duct, Int. J. Numer. Methods Engrg. 28 (1989) $445-459$.

[18] Z. Demendy, T. Nagy, A new algorithm for solution of equations of MHD channel flows at moderate Hartmann numbers, Acta Mech. 123 (1997) 135-149. 